University of Nebraska - Lincoln

DigitalCommons@University of Nebraska - Lincoln

20th \& 21st Century French and Francophone Modern Languages and Literatures, Department Studies International Colloquium

\title{
Un regard écopoétique sur la Terre: évolutions du paysage français dans la photographie contemporaine
}

Anaïs Belchun

Université de Toulouse - Jean-Jaurès, contact@anaisbelchun.com

Follow this and additional works at: https://digitalcommons.unl.edu/ffsc2020

Part of the Comparative Literature Commons, French and Francophone Literature Commons, and the Other French and Francophone Language and Literature Commons

Belchun, Anaïs, "Un regard écopoétique sur la Terre: évolutions du paysage français dans la photographie contemporaine" (2020). 20th \& 21st Century French and Francophone Studies International Colloquium.

27.

https://digitalcommons.unl.edu/ffsc2020/27

This Article is brought to you for free and open access by the Modern Languages and Literatures, Department of at DigitalCommons@University of Nebraska - Lincoln. It has been accepted for inclusion in 20th \& 21st Century

French and Francophone Studies International Colloquium by an authorized administrator of DigitalCommons@University of Nebraska - Lincoln. 
archives-ouvertes

\title{
Un regard écopoétique sur la Terre: évolutions du paysage français dans la photographie contemporaine
}

\author{
Anaïs Belchun
}

\section{To cite this version:}

Anaïs Belchun. Un regard écopoétique sur la Terre: évolutions du paysage français dans la photographie contemporaine. "Parler la Terre" 20th and 21st centuries French and francophone studies International colloquium, Mar 2020, Lincoln, Nebraska, États-Unis. hal-02543769

\section{HAL Id: hal-02543769 \\ https://hal.archives-ouvertes.fr/hal-02543769}

Submitted on 15 Apr 2020

HAL is a multi-disciplinary open access archive for the deposit and dissemination of scientific research documents, whether they are published or not. The documents may come from teaching and research institutions in France or abroad, or from public or private research centers.
L'archive ouverte pluridisciplinaire HAL, est destinée au dépôt et à la diffusion de documents scientifiques de niveau recherche, publiés ou non, émanant des établissements d'enseignement et de recherche français ou étrangers, des laboratoires publics ou privés. 
Pour le colloque « Parler la Terre »

20th and 21st centuries French and francophone studies International colloquium

March, 2020, University of Nebraska-Lincoln (USA)

\title{
Un regard écopoétique sur la Terre : évolutions du paysage français dans la photographie contemporaine
}

\author{
Anaïs Belchun \\ LARA-SEPPIA (Université Toulouse - Jean Jaurès, France)
}

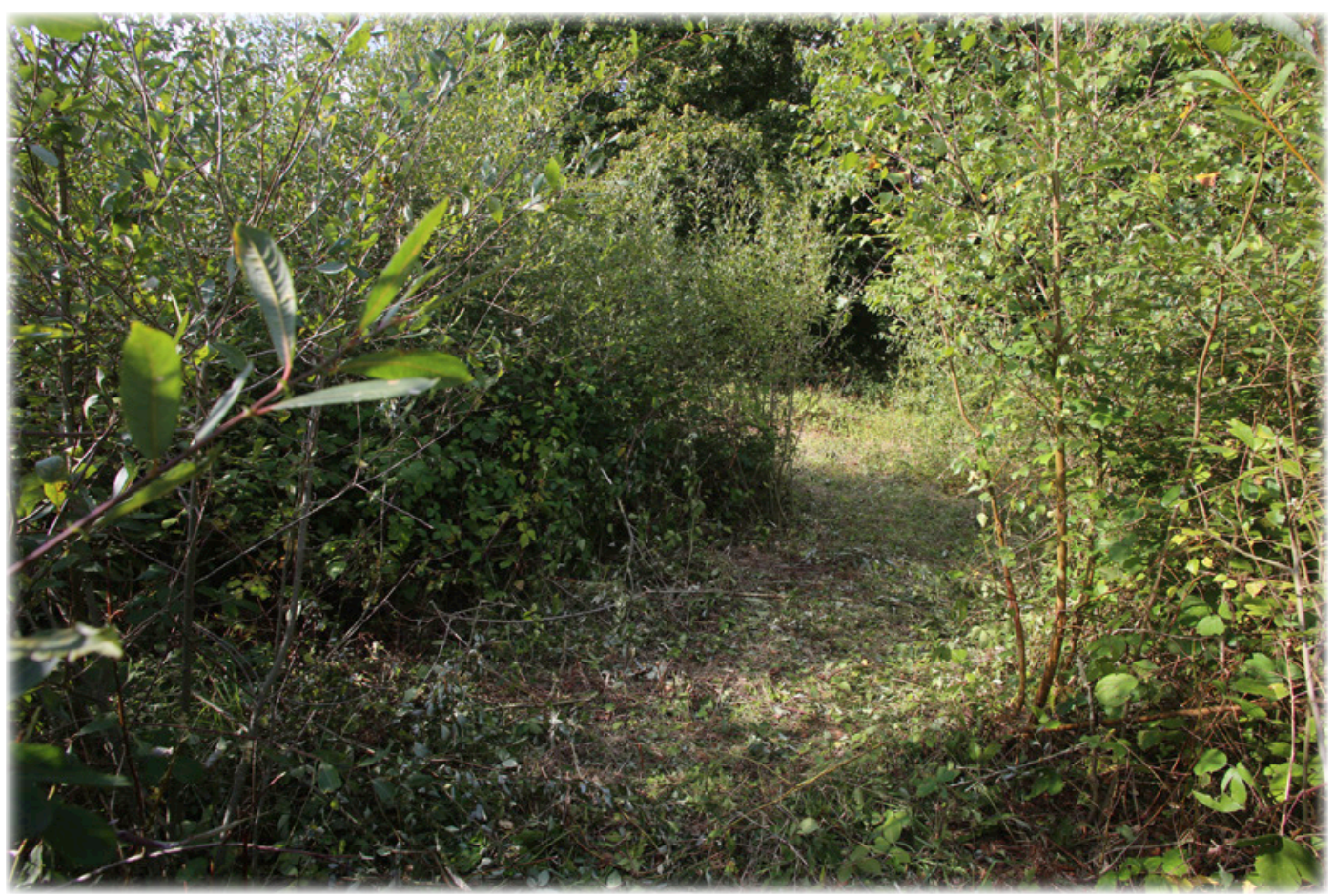

Graphiste, artiste et doctorante en Arts plastiques, Anaïs Belchun prépare une thèse intitulée «Écopoétique paysagère : une pensée de l'écologie à l'œuvre dans les arts du paysage » sous les directions de Patrick Barrès et Sophie Lécole Solnychkine, au sein du LARA-SEPPIA (Université Toulouse - Jaurès). Elle étudie les pensées et pratiques de l'écologie et du paysage selon une approche transdisciplinaire, avec un objectif pédagogique. Sa démarche associe recherche scientifique et création artistique, autour d'un travail de terrain (enquête paysagère, sentier paysager, ateliers d'écoformation) et hors site (photographie, carnets d'artistes, expositions). Elle a organisé plusieurs manifestations scientifiques et artistiques sur le paysage et l'écologie et contribue activement à la revue Gradalis, carnets de topoïétique.

www.anaisbelchun.com 
Nos relations à la Terre, comme ses représentations, évoluent depuis quelques décennies avec le développement d'une pensée et d'une sensibilité écologique. Cela entraine une redéfinition de notre manière de percevoir et de représenter la Terre, sur les plans esthétiques et poétiques. À l'échelle locale (the land), l'esthétique environnementale redéfinit notre relation au milieu comme une relation sensible dans un processus immersif, à travers laquelle les distinctions entre sujet et objet, homme et environnement s'estompent au profit d'une expérience esthétique de la relation. Au niveau global (the earth) se déploie une approche cosmologique qui dépasse la distinction entre Nature et culture, au profit d'une vision de la Terre comme système naturel-culturel et milieu de vie commun à l'ensemble du Vivant. Cet article interroge la manière dont cette évolution s'exprime dans la photographie contemporaine, à travers le cas des paysages français, des années quatre-vingt à aujourd’hui.

\section{Photographie de paysage et relation à la nature : introduction historique}

Si la photographie de paysage n'est plus considérée aujourd'hui comme image objective du monde, elle traduit tout de même la vision que nous en avons. L'évolution des représentations paysagères est donc liée à celle de notre relation à la nature. Dès l'origine de la photographie au début du XIX siècle, le paysage est un thème majeur, relevant d'abord d'une approche documentaire, puis artistique. Dans les deux cas, les premiers paysages reflètent une vision de la nature héritée de l'époque classique et de la Renaissance. La nature est perçue d'un point de vue extérieur, comme un ensemble d'objets étalés dans l'espace, que les scientifiques étudient, tandis que les artistes en représentent les éléments les plus rares, beaux ou impressionnants. Ce qui se traduit dans une représentation paysagère figurative, frontale et distanciée.

Le développement des voyages et du tourisme ouvre ensuite la photographie paysagère au grand public, avec des représentations illustratives qui culminent dans l'invention de la carte postale, reprenant les codes de la peinture des siècles précédents : paysages bucoliques ou pittoresques. Le paysage, offert comme point de vue privilégié, met alors en scène une nature humanisée et idéalisée. Puis l'expansion urbaine fait émerger l’idée nostalgique d'une nature originelle, sauvage et sacralisée. Cette vision s'incarne dans le rêve américain avec la découverte des grands espaces de l'Ouest. Une nouvelle esthétique paysagère s’impose, le sublime, nourrie de cette dévotion aux caractères grandioses de la nature. Les esthétiques pittoresques et sublimes s'y renouvellent ensuite en lien avec la création des parcs naturels nationaux.

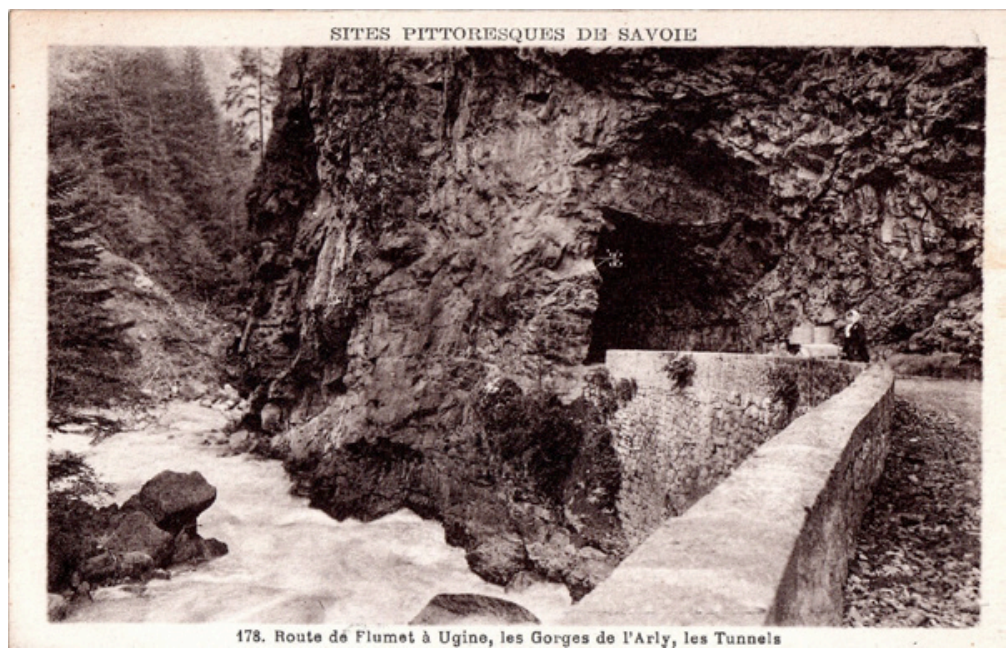

Paysage pittoresque :

Carte postale ancienne, "Sites pittoresques de Savoie » $n^{\circ} 178$

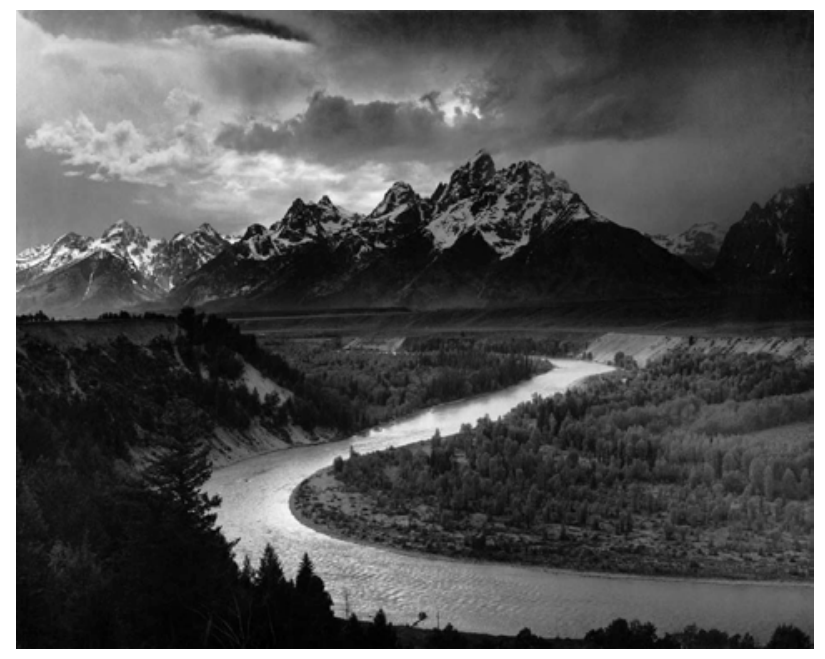

Paysage sublime :

Ansel Adams, The Tetons and the Snake River, Wyoming, 1942 


\section{La fiction verte}

$\mathrm{Au} \mathrm{XX}^{\mathrm{e}}$ siècle, l'écologie provoque à son tour d'importantes évolutions dans notre vision de la nature. Le développement de la science écologique, qui se définit comme l'étude des relations entre les êtres vivants et leur milieu de vie, fait apparaître de nouvelles problématiques et de nouveaux modes de pensée. On peut repérer dans les discours et les représentations associées à une pensée de l'écologie deux axes, qui se distinguent par une vision du monde, un positionnement éthique, des esthétiques et des pratiques différentes - suivant globalement la distinction établie par Arne Naess entre « écologie superficielle » et « écologie profonde » ${ }^{1}$.

Les pensées de l'écologie superficielle sont fondées sur une approche environnementale, anthropocentrée et utilitariste de la nature, associée au modèle du développement durable. Je nomme « fiction verte » l'ensemble des discours et représentations qui en émergent, car il s’agit généralement de représentations simplistes dans lesquelles l'idée de nature est réduite à la végétation, voire à la couleur verte. Cette approche repose sur une vision idéalisée de la Nature (en opposition à la Culture). Il en résulte un ensemble vague de représentations se rapportant indistinctement à la terre, à la nature, à l'environnement ou à l'écologie, qui n'est pas représentatif de la diversité et de la complexité écologique. Il constitue seulement un effet-écologie significatif, qui s’érige en récit sociétal : la « fiction verte ».

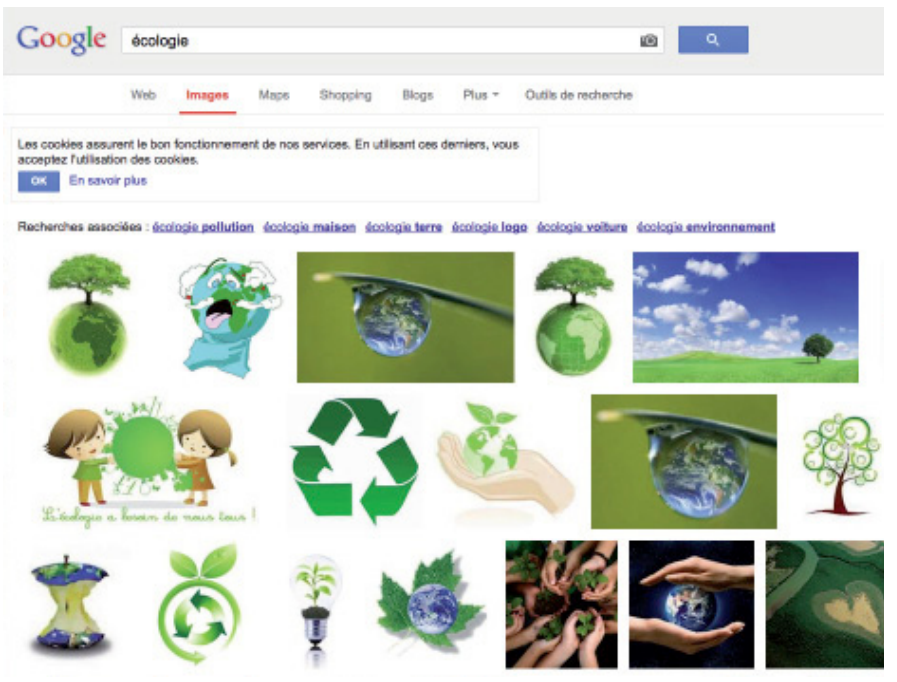

Images caractéristiques de la fiction verte : Recherche du terme « écologie » dans Google Images (2013)

Celle-ci s’exprime dans la photographie avec des « clichés » de paysages naturels, dans les deux sens du terme : une image photographique, ou une idée banale exprimée dans des termes stéréotypés. Les publicitaires utilisent par exemple des représentations paysagères directement héritées des modèles esthétiques du XIX siècle : bucolique (la prairie d'herbe verte avec un seul arbre, sous un ciel toujours bleu) ou sublime (forêt vierge, montagne enneigée, coucher de soleil en bord de mer, etc.). L'évocation de la nature permet de créer un effet-écologie, utilisé comme argument de vente ou de persuasion. Mais ces représentations fictionnelles ne sont que les lieux communs partagés de la fiction verte.
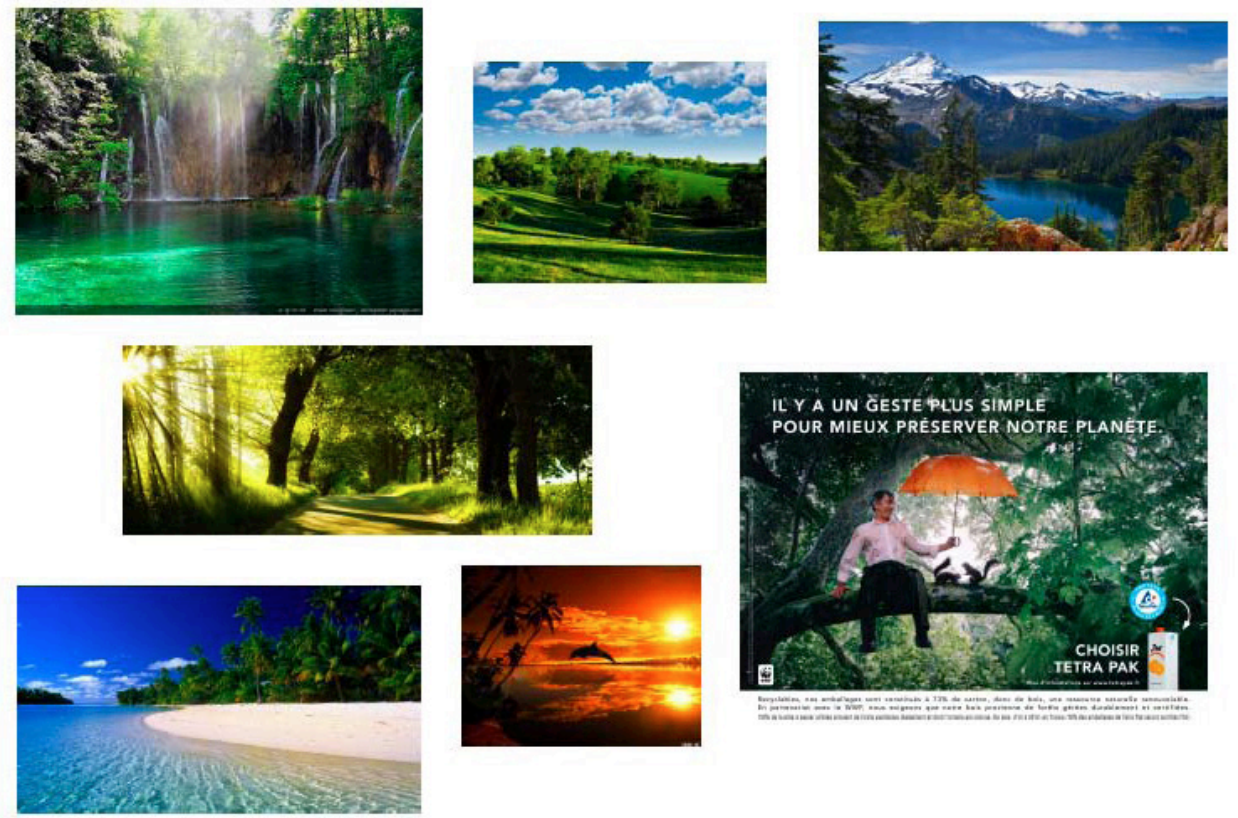

Queslques exemples de fiction verte paysagère

1 Arne NAESs, « Le mouvement d'écologie superficielle et le mouvement d’écologie profonde de longue portée : Une présentation », in Éthique de l'environnement : Nature, valeur, respect, Vrin, 2007, pp. 51-60. 


\section{Le paysage-spectacle}

Un exemple emblématique de cette fiction verte peut être identifié dans les photographies de La Terre vue du ciel $^{2}$ de Yann Arthus-Bertrand, livre réalisé dans l'intention de « témoigner de la beauté du monde et tenter de protéger la Terre ». On remarquera que dans cette série photographique, "l'image aérienne reste une fascination de surface (...) : la Terre n’est qu'un prétexte à faire de belles images ${ }^{3}$. Le discours moralisateur du film est ainsi neutralisé par cette esthétisation, présentant le paysage comme un spectacle. Cette esthétique du paysage-spectacle cache surtout des contradictions importantes entre le discours écologiste revendiqué et la vision du monde véhiculée par ces images. D’abord, ces images entretiennent la représentation illusoire d'une nature sauvage, préservée, et contribuent donc à masquer l'impact réel des activités humaines sur l'ensemble des écosystèmes, à l'échelle planétaire. Cette problématique semble être compensée par Yann Arthus Bertrand par ses images de paysages saccagés, ruinés par les activités humaines (notamment industrielles). Ces paysages-catastrophes représentent ainsi l'envers du décor, sans sortir de ce modèle spectaculaire, à l'instar des films-catastrophes américains. Mais surtout, le point de vue reste le même. La posture extérieure et dominante du spectateur face au paysage vu du ciel reproduit le schéma de l'homme tout-puissant, dominant la Nature. On peut l'interpréter comme un témoignage de l'hubris caractéristique de la société industrielle - or celle-ci se trouve à l’origine de la crise écologique. En contradiction avec le message écologiste qui les accompagne, ces photographies perpétuent donc une vision illusoire de la nature et une prise de distance maximale entre l'homme et son environnement. On peut aussi relever le caractère anthropocentriste, ethnocentriste (voire post-colonial) de ces images.

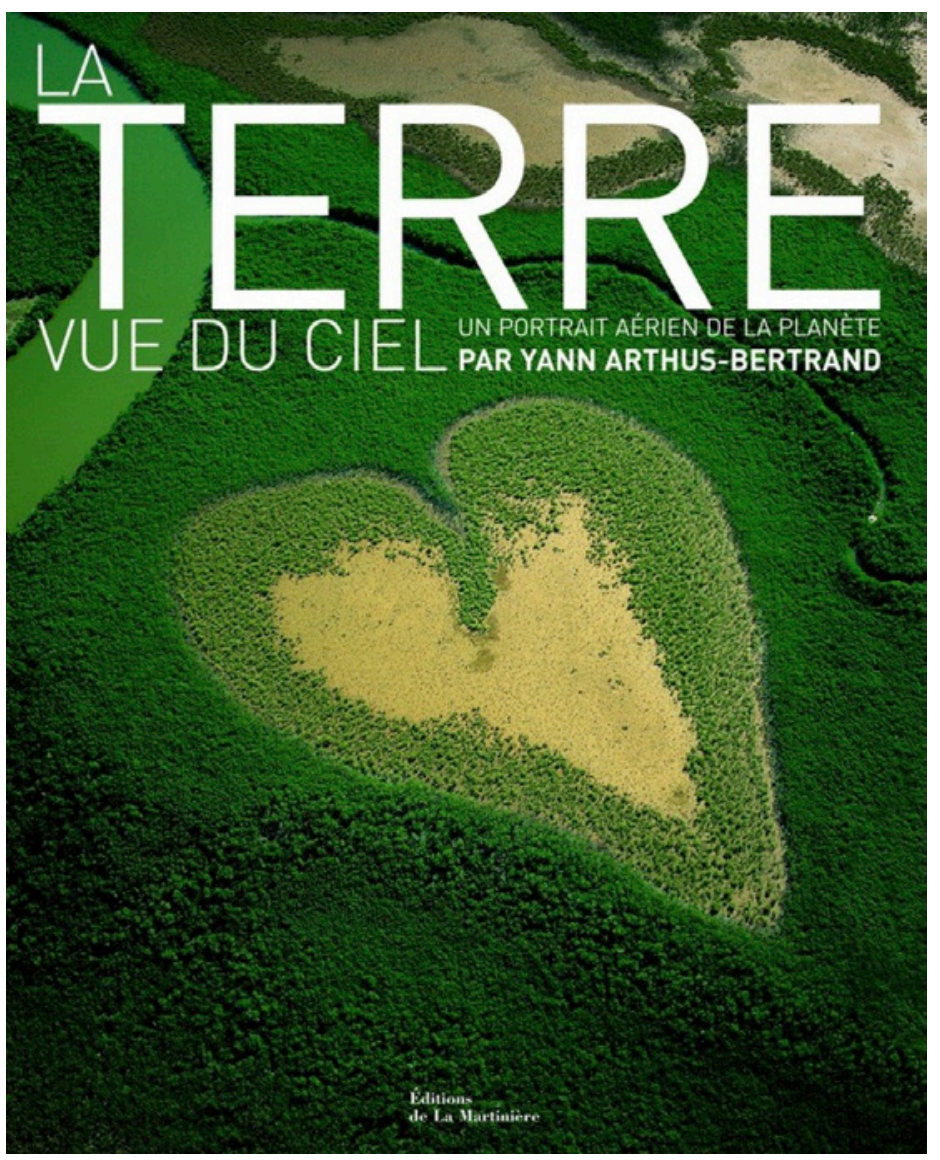

Couverture du livre de Arthus-Bertrand Yann, La Terre vue du ciel: un portrait aérien de la planète, Paris, Éditions de la Martinière, 2012.
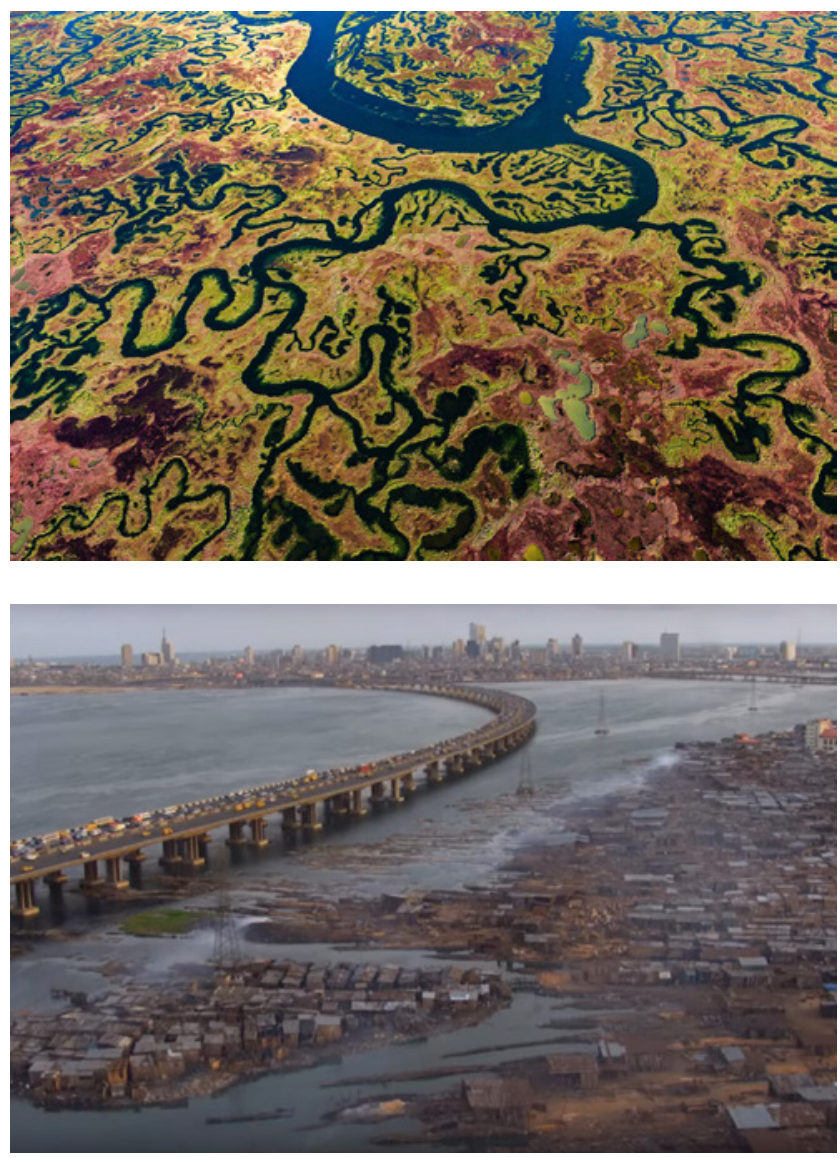

Images extraites du film de Yann Arthus-Bertrand, Home, 2019.

2 Yann Arthus-Bertrand, La Terre vue du ciel : un portrait aérien de la planète, Paris, Éditions de la Martinière, 2012.

3 Estelle BAyon, Vers une esthétique de l'humilité : Visions du monde en présence de la terre dans les cinémas contemporains,

Thèse de Doctorat en Arts et Sciences de l’Art - Cinéma et Audiovisuel, Université Paris I - Panthéon Sorbonne, Paris, 2012. 
La photographie peut aussi servir à remettre en question ces représentations idéalisées d'une nature supposée sauvage. La série d'Emmanuelle Blanc ${ }^{4}$ intitulée "Cartographie d'une extrême occupation humaine " présente ainsi des images mettant en tension le modèle du paysage-spectacle et de la nature sauvage avec la réalité d'un territoire occupé par les humains. Elle photographie d'une manière assez classique des paysages de montagne, apparemment sauvages voire inaccessibles. L'occupation humaine de ces territoires, invisible au premier regard, est révélée par un détail artificiel dans le paysage : la ligne électrique qui traverse le coin gauche de cette image, ou le bâtiment caché sur cette crête rocheuse. Ces accrocs dans le paysage constituent ainsi des accroches ouvrant vers une autre lecture de ces images.

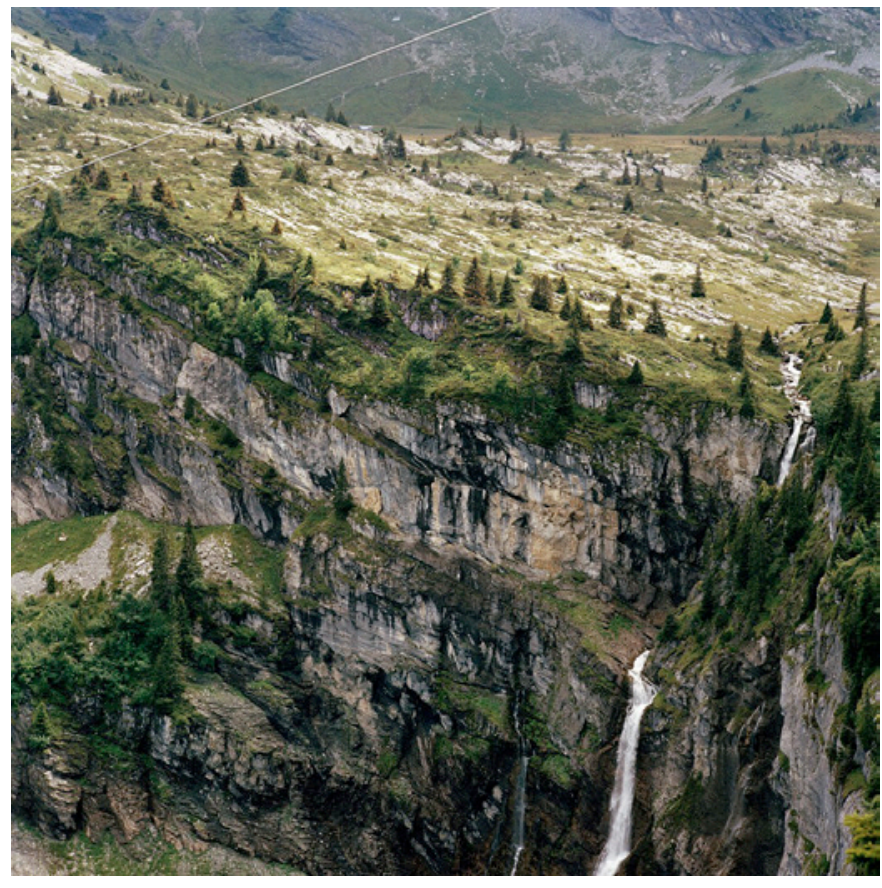

Emmanuelle Blanc, série « Cartographie d'une extrême occupation humaine ", $\mathrm{n}^{\circ} 6$ Altitude $1780 \mathrm{~m}$

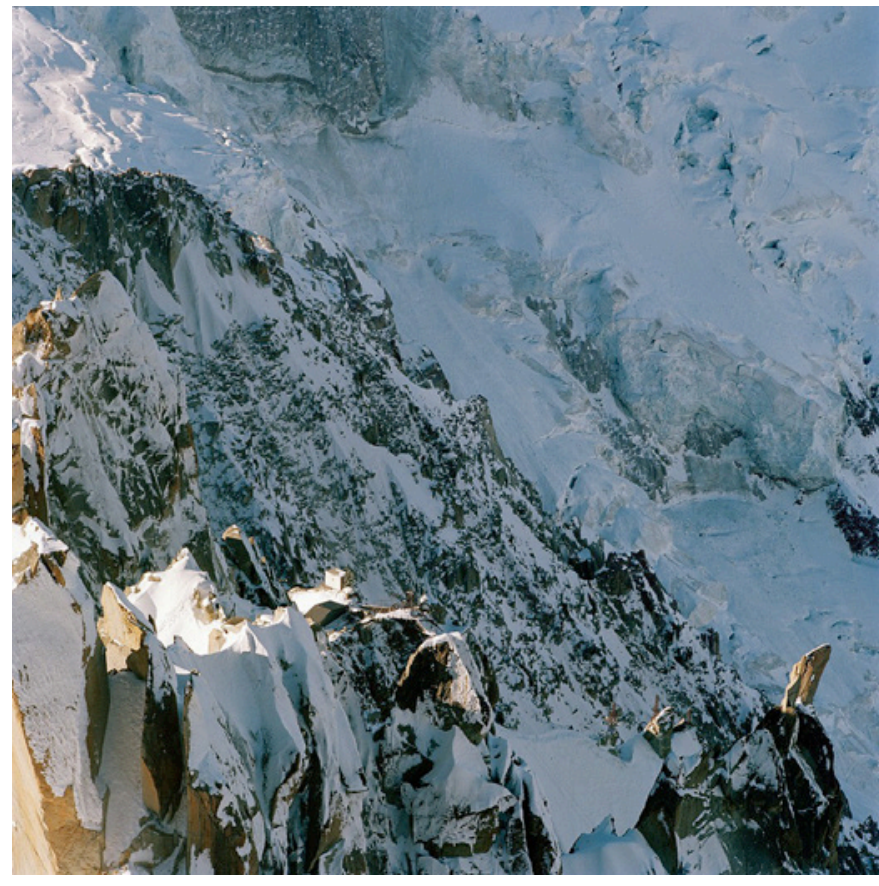

Emmanuelle Blanc, série « Cartographie d'une extrême occupation humaine ", $\mathrm{n}^{\circ} 3$ Altidude $3842 \mathrm{~m}$

Jean-Loup Trassard ${ }^{5}$ reprend avec ironie les clichés de paysages exotiques dans sa série de micro-paysages (exposée sous le nom " Carnets d’un voyageur » et publié dans un livre intitulé " Le voyageur à l'échelle »). Il présente un paysage désertique, dessiné par une trace et trois tas de terre, peut-être dans la cour d’une ferme, ou encore un faux temple maya réalisé avec une série de brindilles et une souche d'arbre. Cette série remet en question notre attrait pour des paysages exotiques en révélant la part de rêve et de fantasme qui les constituent, tout en proposant une alternative à travers l'attention aux détails matériels de notre environnement proche.
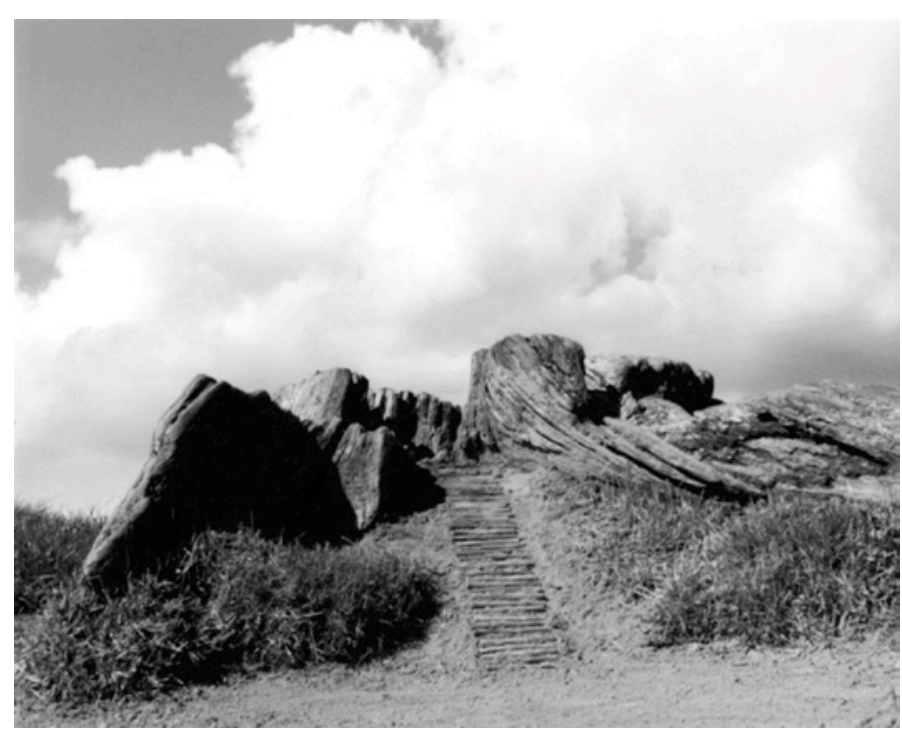

Jean-Loup Trassard, « Ruines d'un temple Maya », dans Le voyageur à l'échelle, Le Temps qu'il fait, 2006.

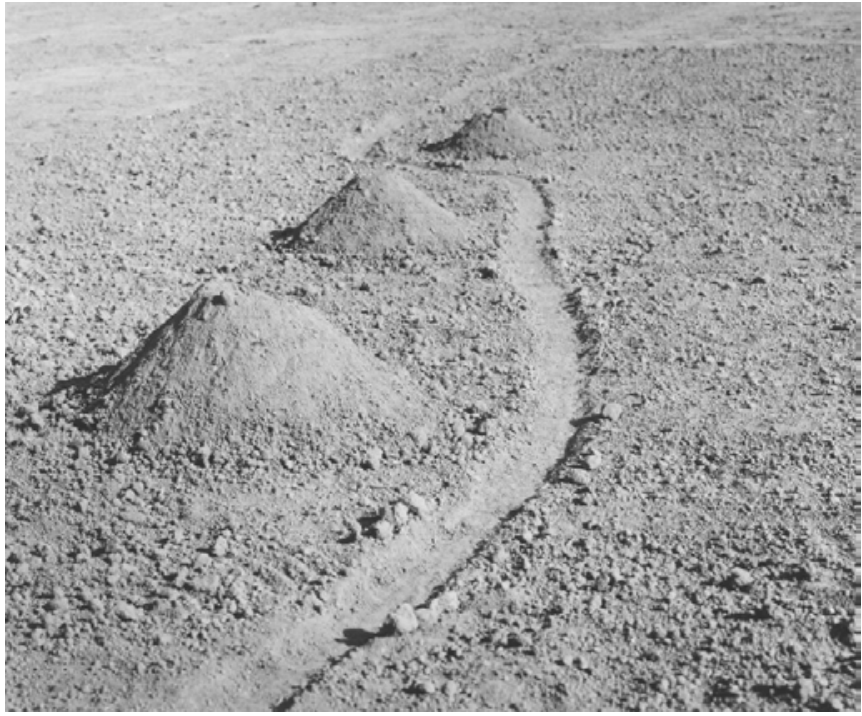

Jean-Loup Trassard, dans Le voyageur à l'échelle, Le Temps qu'il fait, 2006.

4 https://emmanuelleblanc.com/

5 https://www.jeanlouptrassard.com/ 


\section{L’écologie : science, pensée et poétique}

De même que de nouvelles perceptions du paysage émergent dans la photographie, d'autres voies s'inventent dans les pensées de l'écologie, qui se traduisent dans une nouvelle vision du paysage. Ce qu’Arne Naess nomme l'écologie profonde réunit des pensées de l'écologie fondées sur la mise en relation entre l'écologie scientifique et la pensée complexe, réflexions sur l'écologie issues des sciences humaines, et diverses pratiques (agricoles, paysagères, artistiques...). Cette pensée s’articule autour de deux questions : comment habiter la Terre ? et comment vivre dans la communauté du Vivant ? Elle présente aussi une dimension plus sensible, en portant attention à la qualité de notre relation avec notre milieu de vie, ce que je désigne sous le terme d'écopoétique.

L'écologie, en tant que science, concerne « l'étude des relations entre les êtres vivants et leur milieu de vie ». La prise en compte des êtres vivants dans leur ensemble, la notion de « milieu » qui réunit des éléments vivants et non-vivants (caractéristiques géographiques, climatiques, etc.), et surtout l'accent mis sur leurs relations, n’évoquent plus la nature comme un ensemble d’objets figé, mais comme " un système dynamique global, ensemble de processus et de relations ». Ce bouleversement culturel interroge notre rapport au monde et notre manière de l'habiter. Comme le remarque Loïc Fel (dans son ouvrage sur L'esthétique verte ${ }^{6}$ ), face à la nature ainsi redéfinie, « l'expérience esthétique ne se réduit plus à la contemplation frontale d’un ensemble circonscrit, elle s'étend à l'expérience de phénomènes complexes interdépendants » et « se caractérise par l'inclusion du sujet de l'expérience dans la perception d'un ensemble d’interrelations dynamiques ».

L'esthétique paysagère passe alors d'une représentation du lieu, selon un modèle figé et fermé, à une présentation des liens, notamment ceux de l'être humain avec son milieu de vie. Pour reconsidérer le paysage à partir de la notion de milieu, je m’inspire de la mésologie. Ce terme a été créé par Augustin Berque pour désigner la « science des milieux, c'est-à-dire la relation spécifique que tout être vivant crée avec son environnement »". Cette approche mésologique du monde se distingue de l'approche naturaliste, qui ne se préoccupe que des caractéristiques physiques et biologique de l'environnement, qui est le même pour tous. Le milieu au contraire est singulier : il est propre à un organisme ou a une espèce, à une personne ou à une société. La mésologie met donc en avant la subjectivité de la relation qui s’établit entre un être vivant et son milieu de vie.

\section{Le paysage - milieu de vie : territoire habité, pays et paysans}

À la fin du $\mathrm{XX}^{\mathrm{e}}$ siècle, développement urbain, tourisme et prise de conscience écologique font émerger un intérêt nouveau pour le paysage, qui devient « un écrin de vie à préserver ». Les collectivités publiques commandent des missions photographiques visant à observer les dynamiques paysagères, avec des séries de prises de vue par différents photographes étalées dans le temps : les missions de la DATAR ${ }^{8}$ (1983-1989), de l'Observatoire photographique national du Paysage ${ }^{9}$ (depuis 1989) puis de nombreux Observatoires photographiques du paysage ${ }^{\mathbf{1 0}}$ locaux. Révélant une nouvelle vision du paysage intégrant les relations entre l'humain et le milieu, ces représentions paysagères présentent la nature sans l’idéaliser, ni la vider de toute présence humaine. Elles dévoilent le processus de construction des paysages à travers les relations entre le territoire et ses habitants, mettant notamment en avant la dévalorisation paysagère due à l'urbanisation et à l'industrialisation. La subjectivité du regard du photographe est alors revendiquée comme un moyen de présenter le monde tel que nous le voyons au quotidien : des paysages ordinaires.

6 Loïc FEL, L’esthétique verte : de la représentation à la présentation de la nature, Seyssel, Champ vallon, coll. « Pays-paysages », 2009.

7 Augustin Berque, Là, sur les bords de l’Yvette : dialogues mésologiques, Éditions Éoliennes., 2017.

8 https://missionphotodatar.cget.gouv.fr/

9 https://terra.developpement-durable.gouv.fr/observatoire-photo-paysage/home/

10 http://extranet.observatoires-photographiques-paysages.din.developpement-durable.gouv.fr/ 


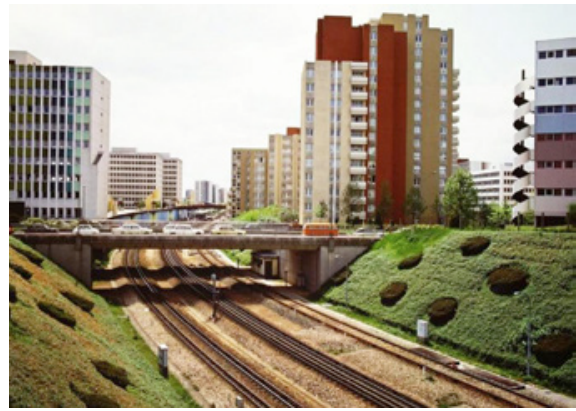

Robert Doisneau, série « Banlieue d'aujourd'hui, dans les banlieues et villes nouvelles de la région parisienne », 1984 (Mission DATAR)

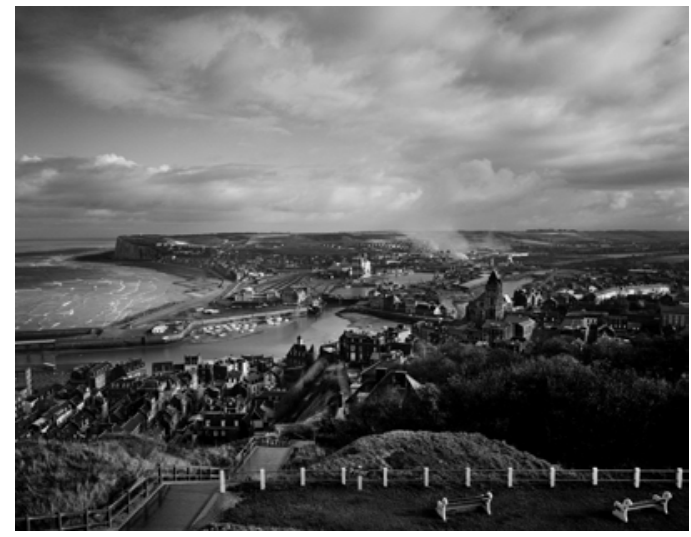

Gabriele Basilico, Le Tréport, série « Bord de Mer », 1985 (Mission DATAR)

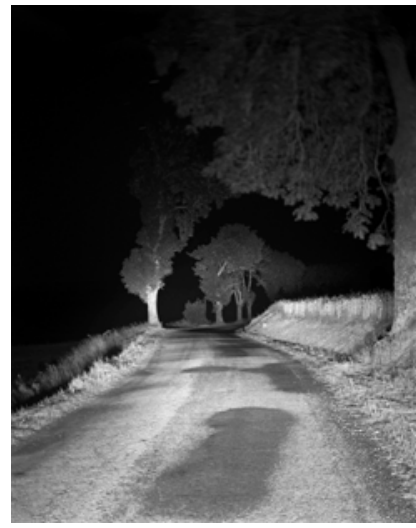

Alain Ceccaroli, série « Paysages de la route, des Alpes aux Pyrénées », 1985 (Mission Datar)

Thibaut Cuisset ${ }^{11}$, inspiré par les images de la DATAR, a adopté une approche lente et contemplative pour présenter les paysages ordinaires des campagnes françaises. Les séries « Une campagne photographique - La Boutonnière du Pays de Bray ${ }^{12}$ et "Campagnes françaises ${ }^{13}$ présentent des paysages aux couleurs douces, sans figure humaine, sans aucun caractère pittoresque ou sublime, mais touchantes par leur simplicité et leur sincérité. On pourrait y voir une mise en œuvre paysagère du neutre de Roland Barthes ${ }^{14}$ ou de la fadeur dont François Jullien fit l'éloge ${ }^{15}$.
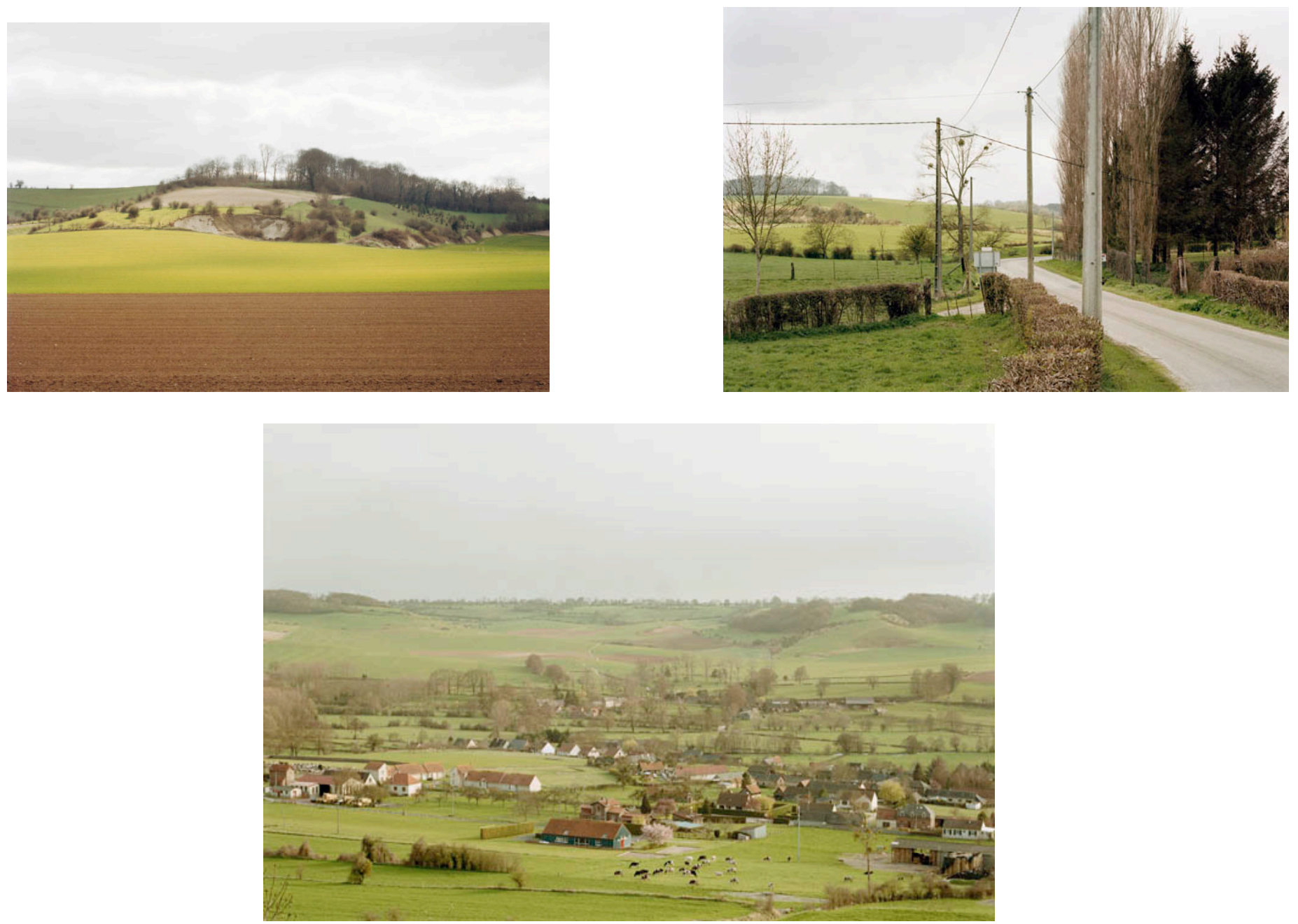

Thibaut Cuisset, Série « Une campagne photographique », 2006

11 https://www.fillesducalvaire.com/artiste/thibaut-cuisset/

12 Thibaut CuIsset, Une campagne photographique - La Boutonnière du Pays de Bray, Filigranes, 2009.

13 Monographie posthume réunissant des photographies prises entre 1958 et 2017 : Thibaut CuIssEt, Campagnes françaises, Steidl, 2019.

14 Roland Barthes, Le neutre, cours au Collège de France (1977-1978), Seuil, 2002. Voir aussi : Lécole-Solnychkine Sophie, Lectures paysagères de la notion de Neutre, Thèse de doctorat en Arts plastiques, Toulouse 2 - Le Mirail, Toulouse, 2009.

15 François Jullien, Éloge de la fadeur, à partir de la pensée et de l'esthétique de la Chine, Le livre de poche, 1993. 
La série photographique de Raymond Depardon « La terre des paysans ${ }^{16}$ est plus représentative du rapprochement entre la photographie de paysage et la photographie humaniste, sous la forme de portraits de paysans photographiés dans leur milieu de vie : la ferme. Elle revalorise une campagne habitée, à travers des paysages construits par la relation entre le pays et les paysans.

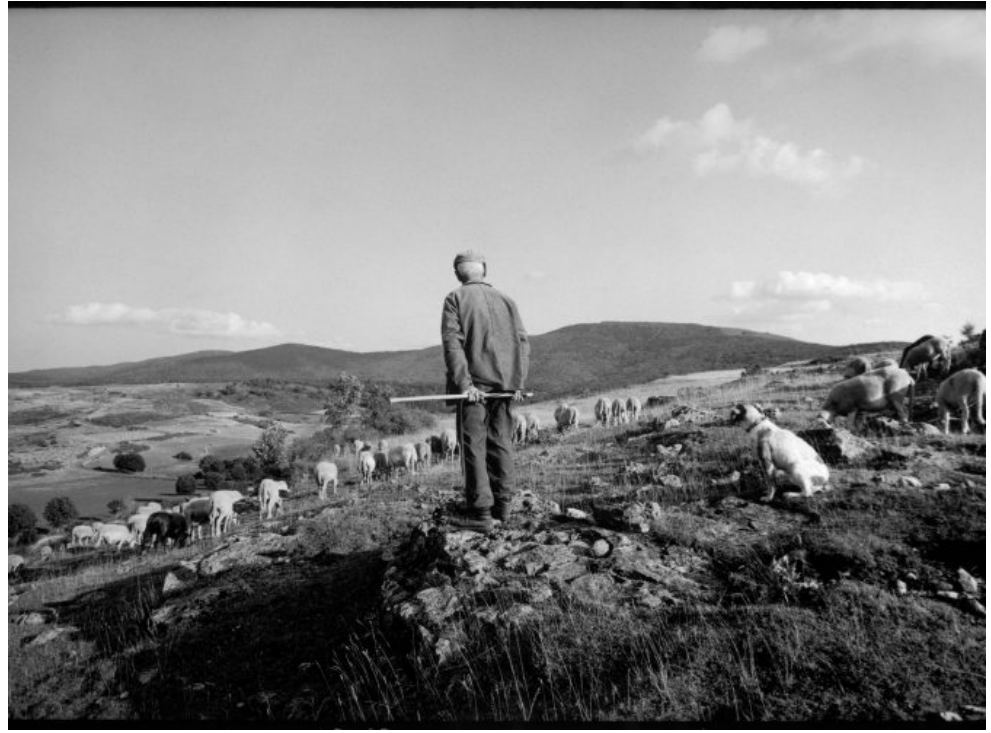

Raymond Depardon, série « La terre des paysans », 1993

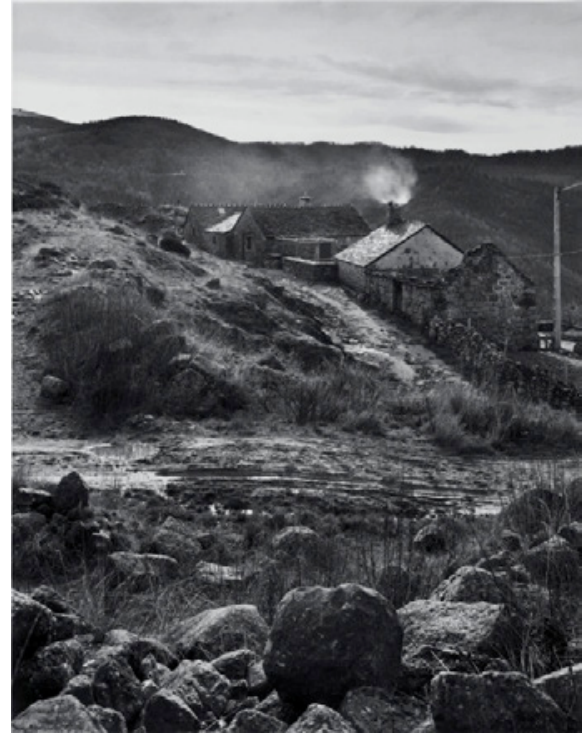

Raymond Depardon, Grizac, Lozère, série « La terre des paysans », 1993

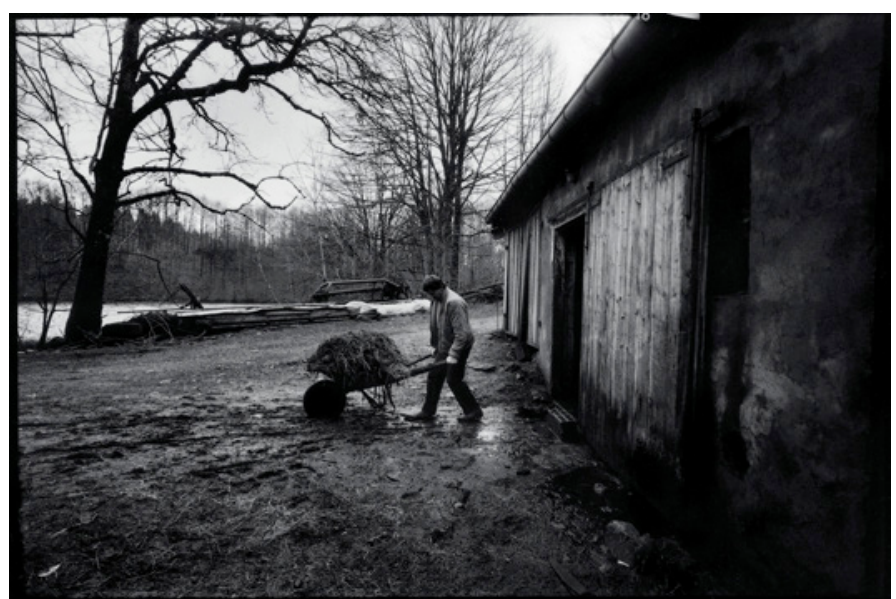

Raymond Depardon, La goutte au fresne, Servance, Franche comté, série « La terre des paysans », 1993

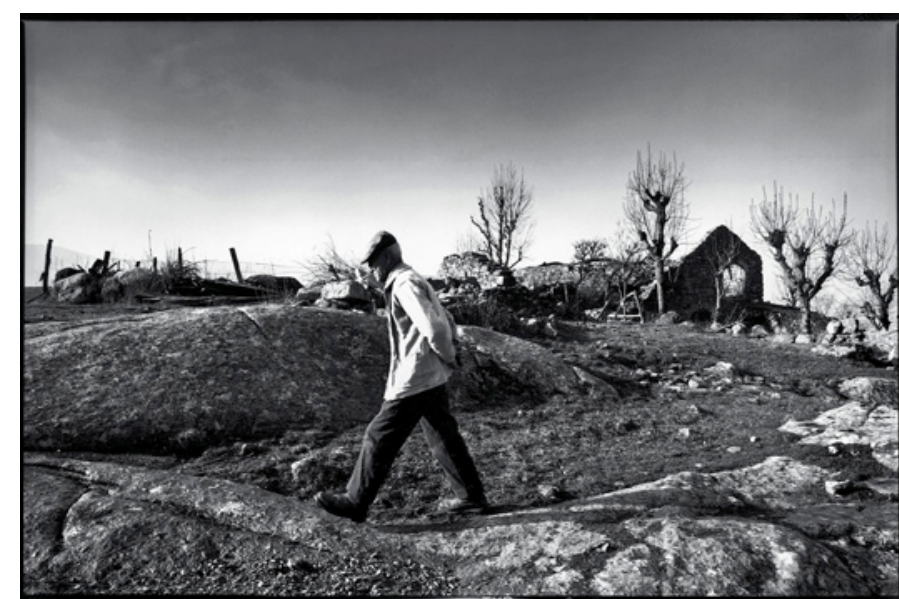

Raymond Depardon, Le Villaret, Lozère, série « La terre des paysans », 1993 
De jeunes photographes perpétuent cette approche du paysage, comme Lise Gaudaire ${ }^{17}$. Dans la série " Paysans, paysages », elle associe le portrait d'un paysan en gros plan avec une photographie de son " paysage préféré ». Le rapprochement esthétique entre un visage et un paysage, et les entretiens associés, révèlent la relation intime entre une personne et un milieu. Dans la série « Les faiseurs de paysages », elle associe des photographies paysagères de friches et de forêts avec des portraits en pied des personnes qui façonnent ces paysages par leur activité quotidienne : bucherons, forestiers de l’ONF, agriculteurs... Ces photographies, accompagnées d'enregistrements sonores et de textes, présentent des espaces ruraux non habités, mais cependant fortement anthropisés et en transformation perpétuelle.
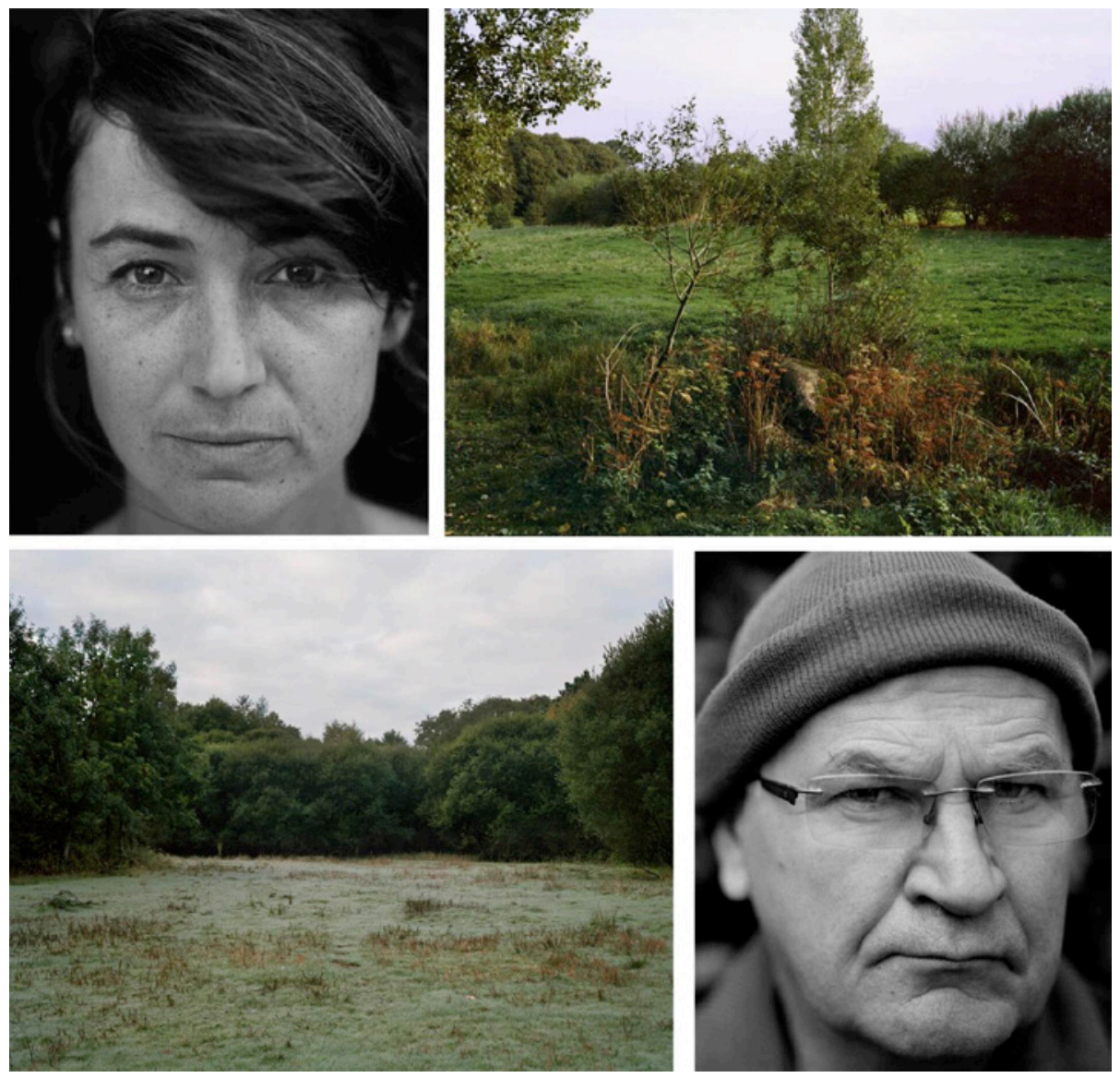

Lise Gaudaire, série « Paysans / Paysages », Creuse / Île-et-Vilaine, 2013-2014
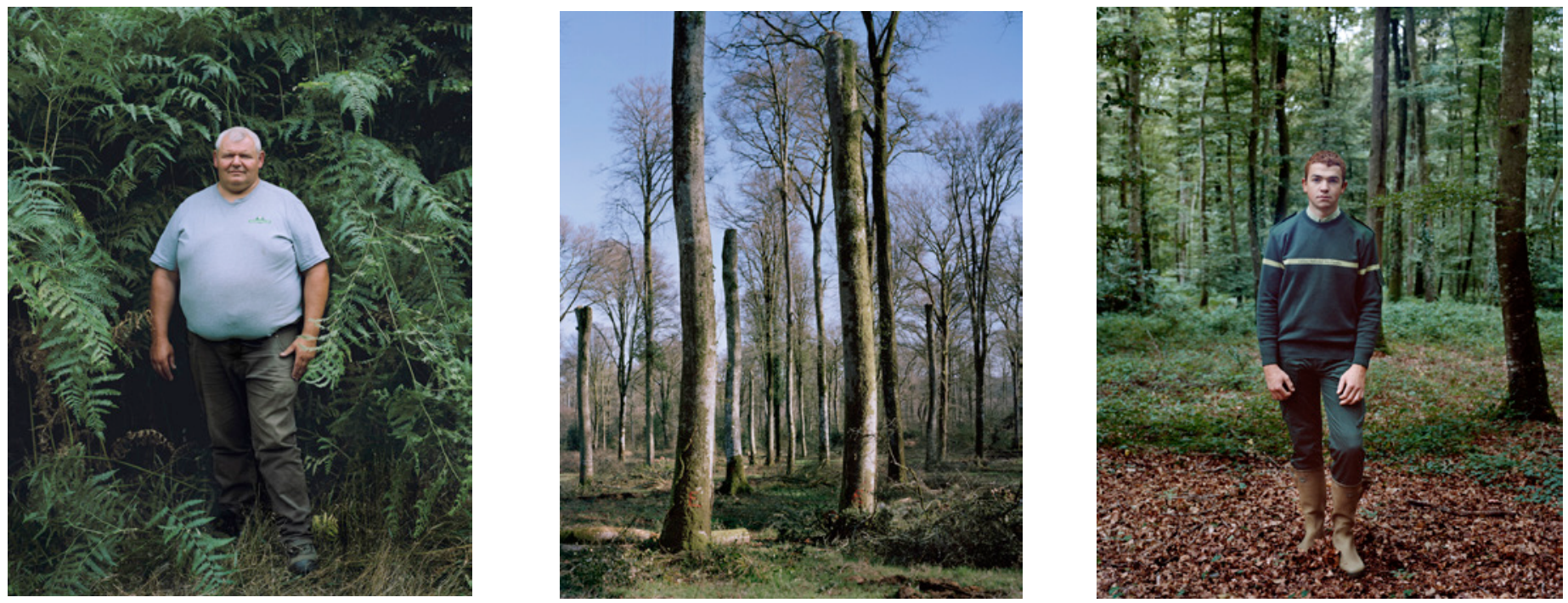

Lise Gaudaire, série « Les faiseurs de paysages, Chapitre 1 : la forêt », Île-et-Vilaine, 2017-2018 


\section{Écologie scientifique et photographie : le paysage entre art et science}

Dans les années quatre-vingt, l'approche photographique du paysage a aussi évolué dans le domaine scientifique, avec le développement en France de l'écologie du paysage. Cette sous-discipline récente de l'écologie est fondée sur des prises de vue aériennes, permettant d’observer les structures spatiales et dynamiques paysagères à une échelle plus vaste que l'écosystème. Ces photographies révèlent les dynamiques entre différents écosystèmes, et la manière dont les interactions entre les éléments naturels, les végétaux, les animaux et les activités humaines structurent les paysages. Ces photographies aériennes sont progressivement complétées par une photographie au sol, notamment à travers la création d’Observatoires Photographiques du Paysage locaux, dédié à l’observation écologique des relations entre les êtres humains et leur milieu de vie.
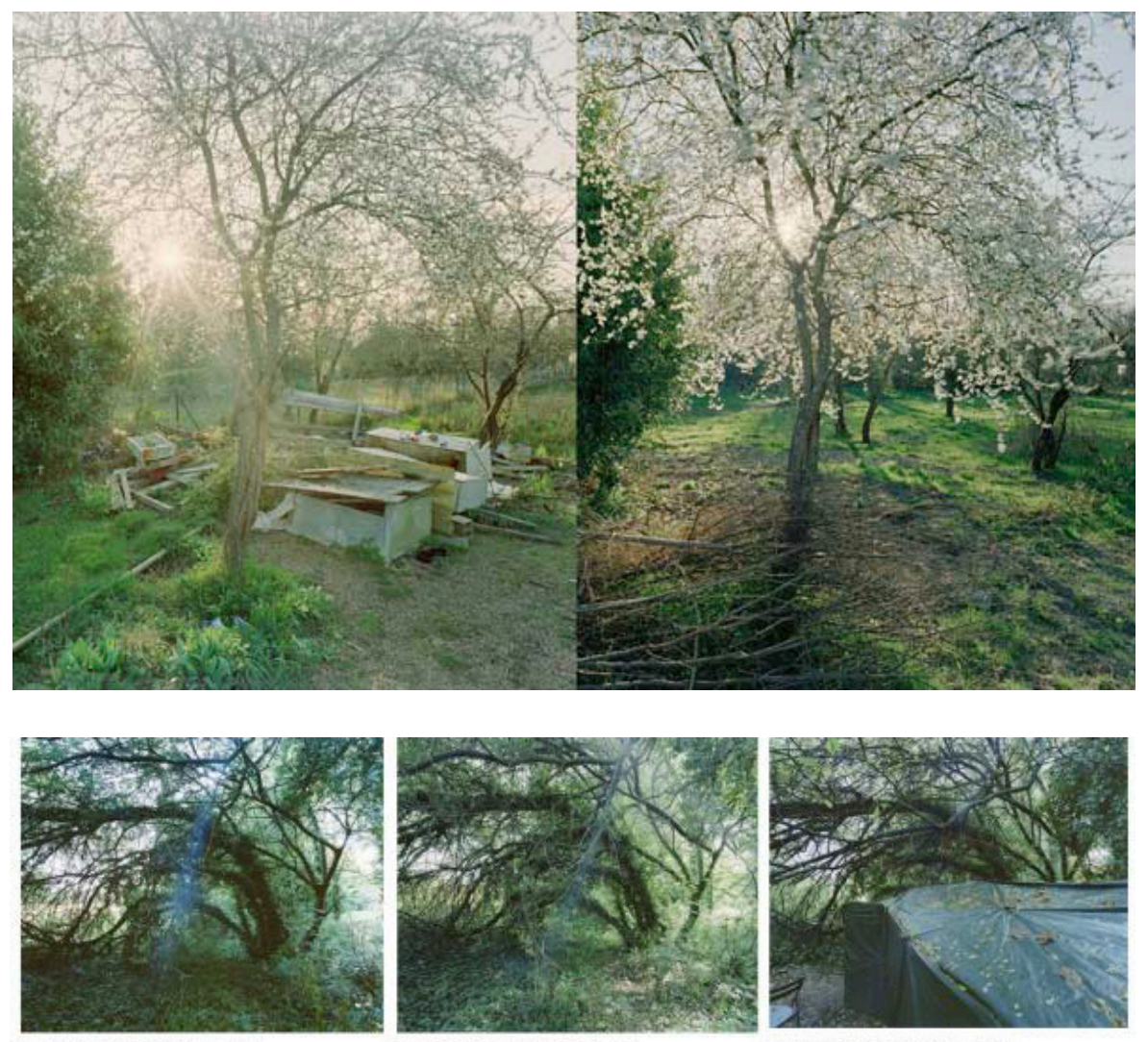

Fig.2Z1SB1/51,23/07/15, 11:52

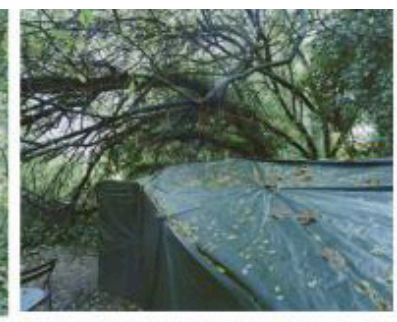

Fig, 1 Z158111], 23/07/14, $09: 30$
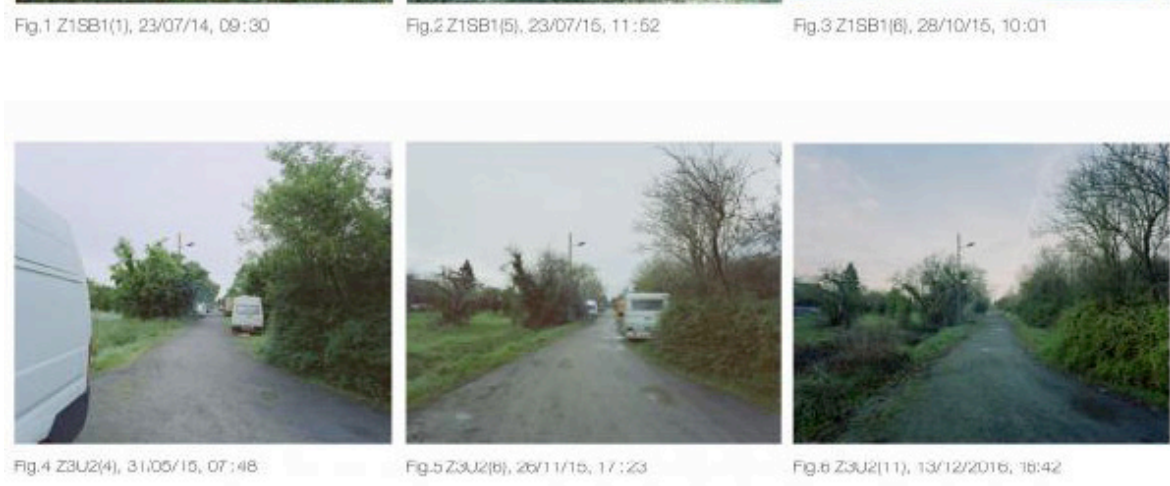

Fig. 4 Zauz/4), 31:00/15, or: 48

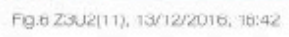

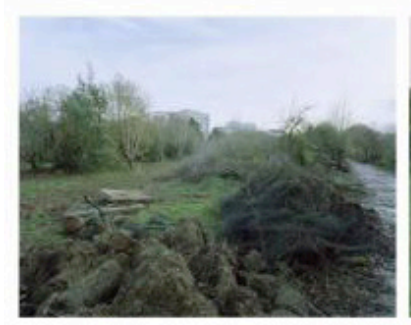

Fig. $722: 12 / 3), 13 / 12 / 14,15: 28$

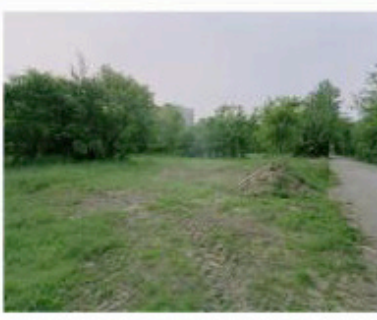

Fig.8 Z2u12,4], 10v05/15, $16: 40$
La photographe Caroline Cieslick a ainsi travaillé à l'Observatoire photographique du paysage des Prairies Saint-Martin à Rennes, au sein de l'équipe de l'observatoire d'écologie urbaine, dans le cadre de l'aménagement de cette friche urbaine en parc. Sur ce site de 24 hectares, elle a réalisé des photographies à la chambres de 24 points de vue, quatre fois par an pendant plusieurs années. Ces images ont montré la dynamique propre à cet espace en friche : cycles saisonniers de la végétation, habitation éphémère (squats, habitat nomade), ruptures (coupes de végétation, aménagements anti-camions), adaptations (reprise de la végétation, déplacements des squatteurs), etc. À ces images sont associées des photographies satellitaires et des captations de données météorologiques (température, précipitations, vent). Ces photographies ont été exposées, mises en page sous la forme de planches d'archives et d'atlas paysager, constituant ainsi des documents pour la recherche en écologie urbaine. 
Cet intérêt écologique pour les friches urbaines se retrouve aussi dans les photographies de Myr Muratet ${ }^{\mathbf{1 8}}$, dans la Flore des friches urbaines ${ }^{19}$ qu'il a réalisée avec Audrey Muratet (écologue et botaniste) et Marie Pellaton (graphiste et illustratrice). Dans cet ouvrage, les photographies, croquis et descriptions botaniques sont complétées par des photographies paysagères des friches. Ces représentations mettent en valeur la dynamique et la diversité écologique de ces milieux, notamment végétale, certains objets ou animaux révélant également l'occupation humaine des lieux (déchets, restes de campement, meute de chiens...). Ces représentations paysagères, en marge du statut documentaire des photographies botaniques, visent à favoriser la découverte de ces milieux, de manière à la fois scientifique et sensible.
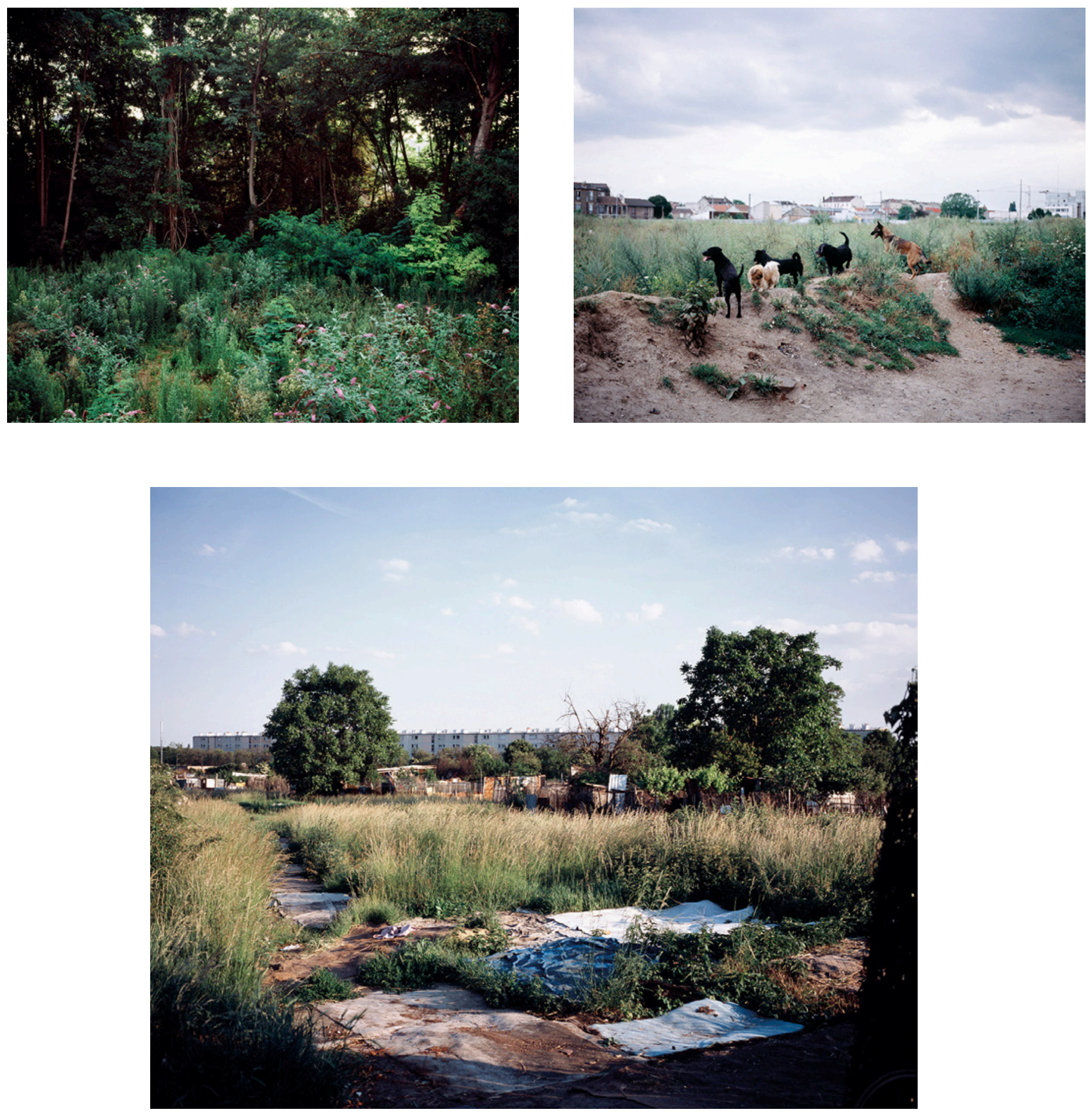

Myr Muratet, Flore des friches urbaines

18 https://www.myrmuratet.com/

19 Audrey Muratet et Myr Muratet, Flore des friches urbaines du nord de la France et des régions voisines, Éditions Xavier Barral, 2017. 


\section{Le paysage écopoétique : une relation sensible}

On devine dans toutes ces images la sensibilité personnelle du photographe au paysage, cependant en retrait par rapport à l'intention documentaire. Un engagement artistique plus affirmé permet à d'autres photographes de mettre en avant la relation singulière et sensible qu'ils entretiennent avec le paysage. Cette relation met en jeu les trois dimensions du sensible : la sensation (corporelle), la sensibilité (émotionnelle, esthétique) et la signification (par l’interprétation des signes).

Christophe Sidamon-Pesson ${ }^{20}$ est un photographe naturaliste, qui prend le temps de découvrir les milieux naturels qu'il photographie, suivant une approche immersive. Ses photographies des milieux sensibles comme les tourbières ou les forêts anciennes révèlent son expérience sensible en immersion profonde, ce qui se traduit par exemple par des photos prises au grand angle, au ras du sol ou à la surface de l'eau. La diversification des points de vue, des focales, des perspectives, constituent de nouveaux modes opératoires, introduits par son approche locale et circonstanciée des milieux. Ces images témoignent de l'attention qu'il porte à l'ensemble des plantes et des animaux qui habitent et composent ces milieux : il montre les cycles écologiques à travers ses photos d'arbres morts servant de support à d'autres formes de végétation ou des différentes saisons, et raconte ses rencontre avec les animaux, comme un chamois caché derrière les feuillages.
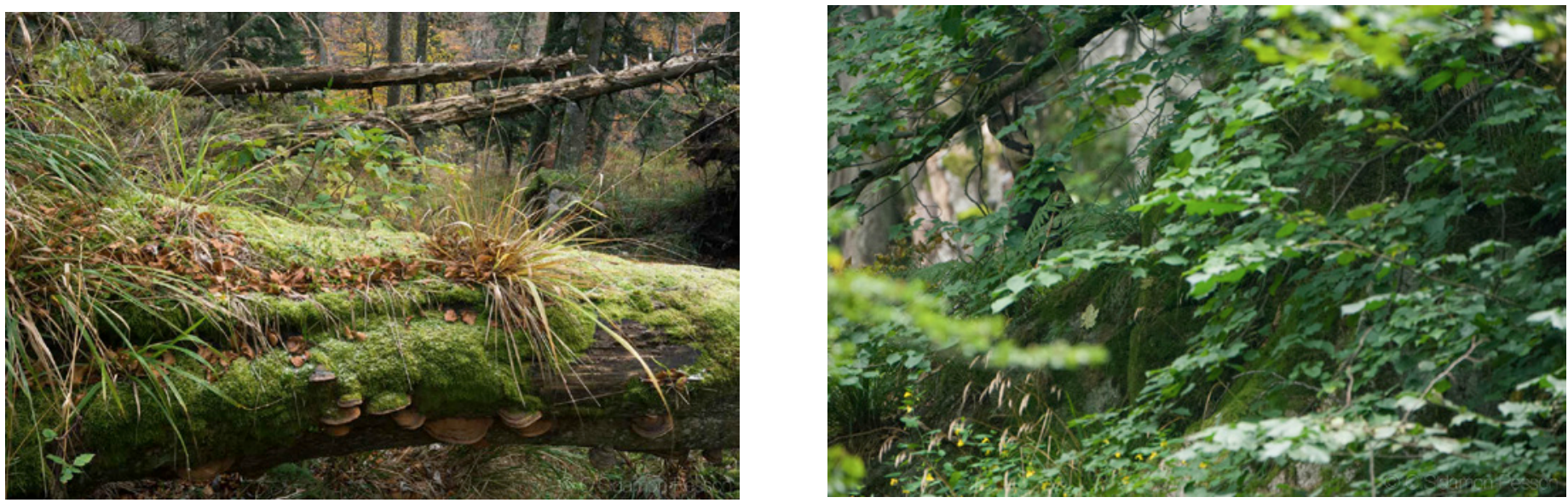

Christophe Sidamon-Pesson, série « Forêts essentielles »

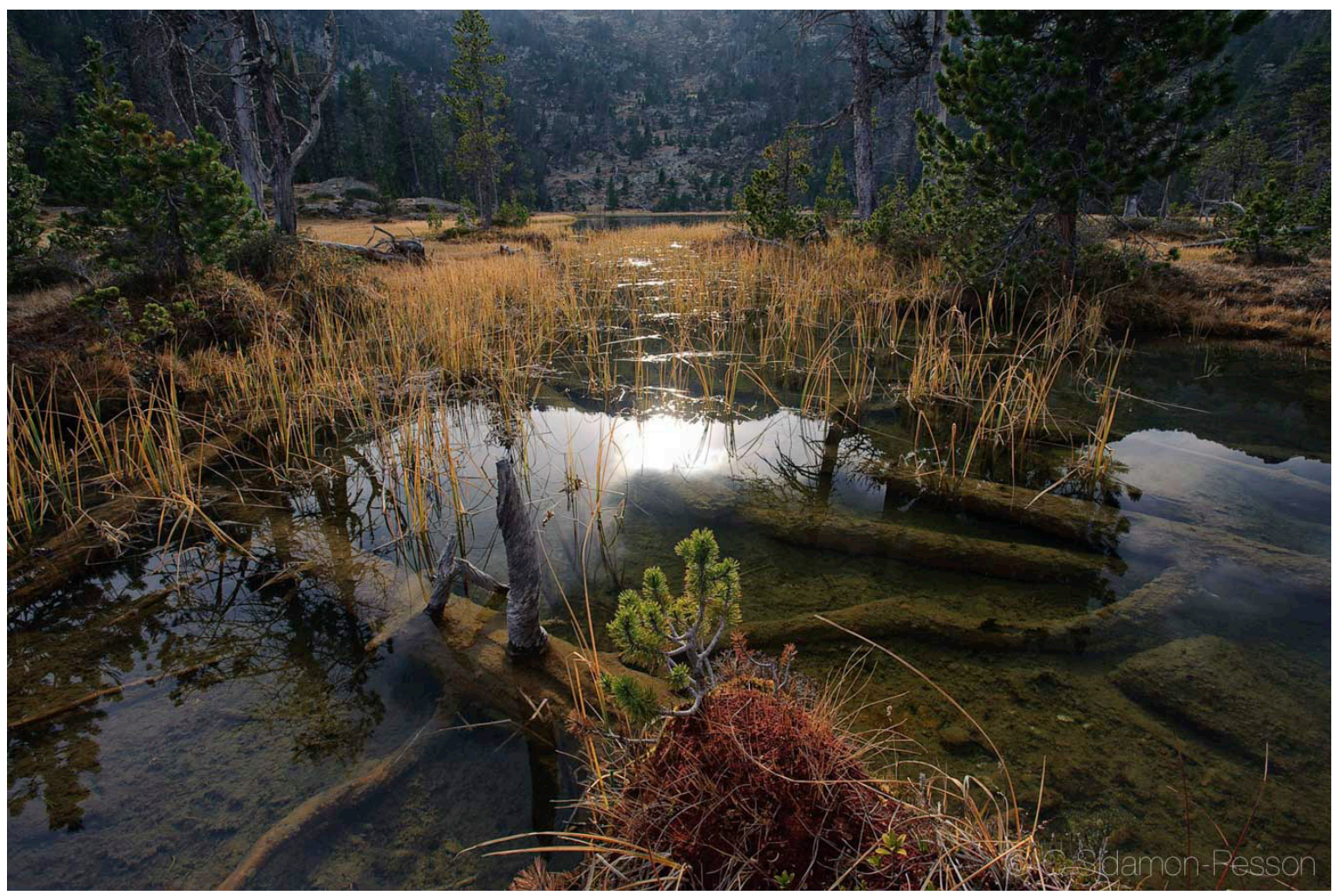

Christophe Sidamon-Pesson, série « Tourbières, un jardin merveilleux »

20 https://www.c-sidamon-pesson.com/ 
L'écrivain et photographe Jean-Loup Trassard ${ }^{21}$ (précédemment cité) exprime son attachement à la campagne mayennaise à travers des photographies en noir et blanc. La présence de la matière, la subtilité des lumières, les vues à ras de sol et l'attention aux traces d'activité agricole expriment sa connaissance intime du milieu, non seulement en tant que paysan, mais aussi en tant que poète. Ces photographies révèlent un paysage connu par cœur et par corps, à travers une fréquentation quotidienne et active. Les écrits accompagnant ces images confirment cette approche, révélant également une attention au vivant sous toutes ses formes : végétal, animal ou humain, domestique ou sauvage, dont les relations complexes s’expriment dans les paysages vécus.

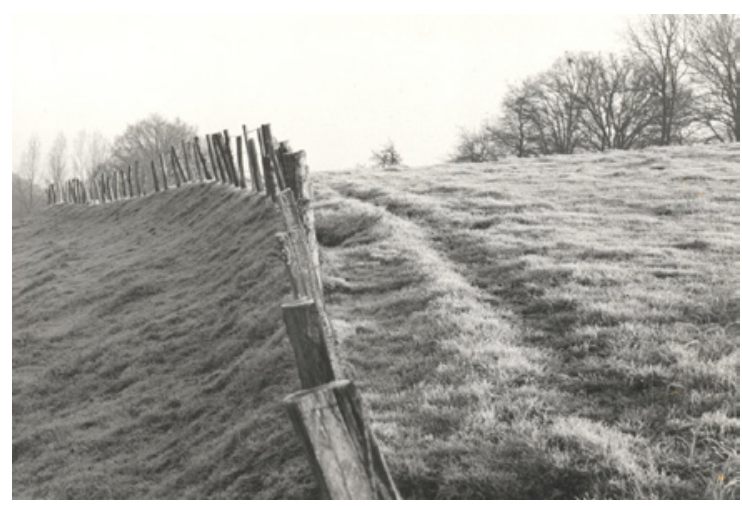

Jean-Loup Trassard, Clôture, série « Territoire »

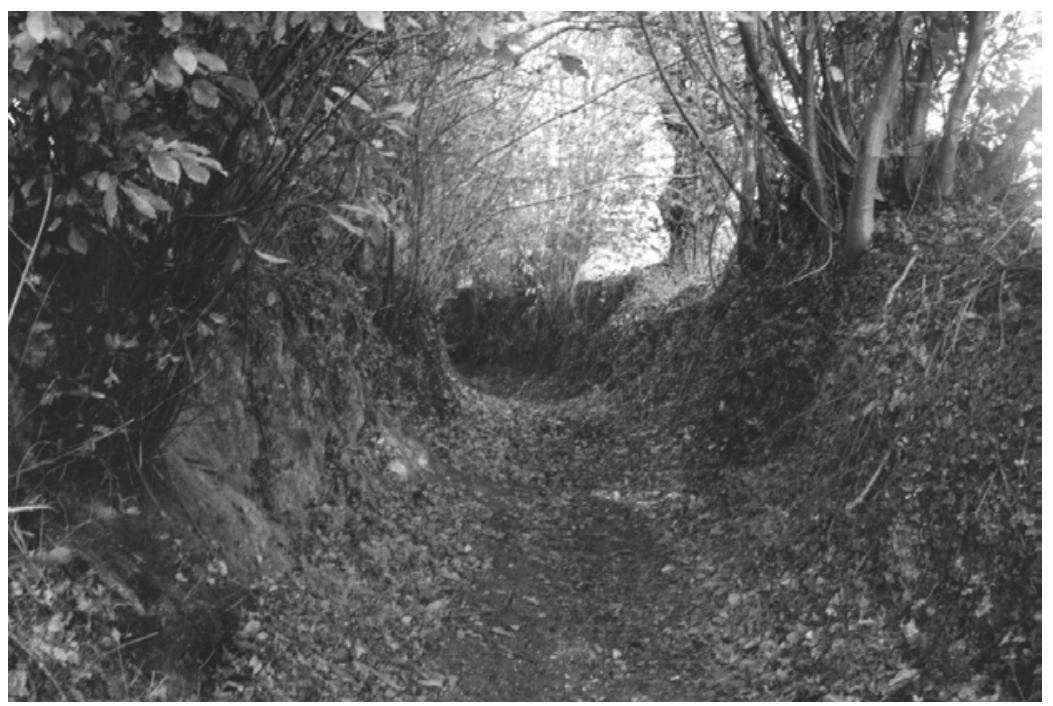

Jean-Loup Trassard, Chemin creux, série « Territoire »

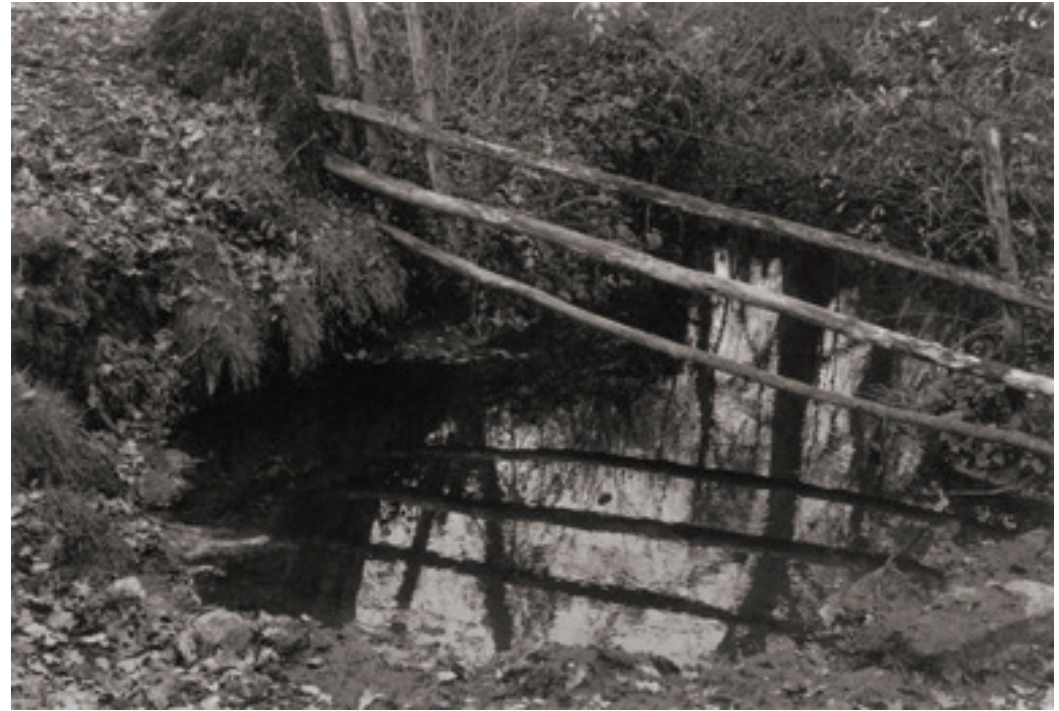

Jean-Loup Trassard, série « Nuisibles »

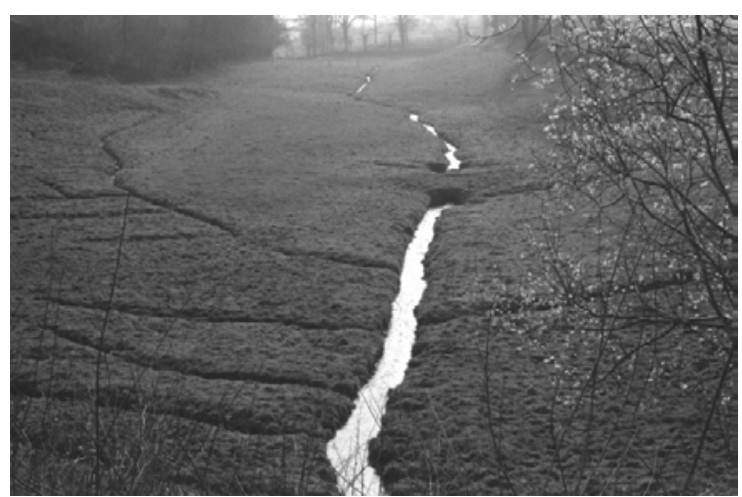

Jean-Loup Trassard, Ruisseau, série « Territoire »

21 https://www.jeanlouptrassard.com/ 
Les artistes Pierre Minot et Gilbert Gormezano ${ }^{22}$ ont mis en scène cette relation à la fois physique et poétique que le corps et l'image tissent entre l'humain et le monde, dans diverses séries photographiques. Ils expérimentent par exemple la manière dont le corps humain, nu, peut se fondre dans un paysage minéral ${ }^{23}$, à travers des compositions où la relation entre le corps et les éléments environnants oscillent entre confrontation et fusion. Dans certaines photographies, le corps humain émerge de la terre et n’y laisse qu'une trace énigmatique $^{24}$, dans d'autres la figure humaine se réduit à une silhouette minuscule, une ombre ou un reflet, perdue dans l'immensité du monde ${ }^{25}$. Enfin dans leurs dernières séries réalisées lors de voyages en bord de mer ${ }^{26}$, c'est par l'écrit (et en couleur) que s'exprime la relation entre l'esprit humain et le paysage qu'il contemple, que le texte soit inscrit directement sur la roche ou imprimé aux côtés des images dans les recueils de voyages. De ces associations de mots et d’images émerge une résonance poétique entre le milieu marin et l'esprit humain, par laquelle le corps, l’esprit et le paysage s’accordent.
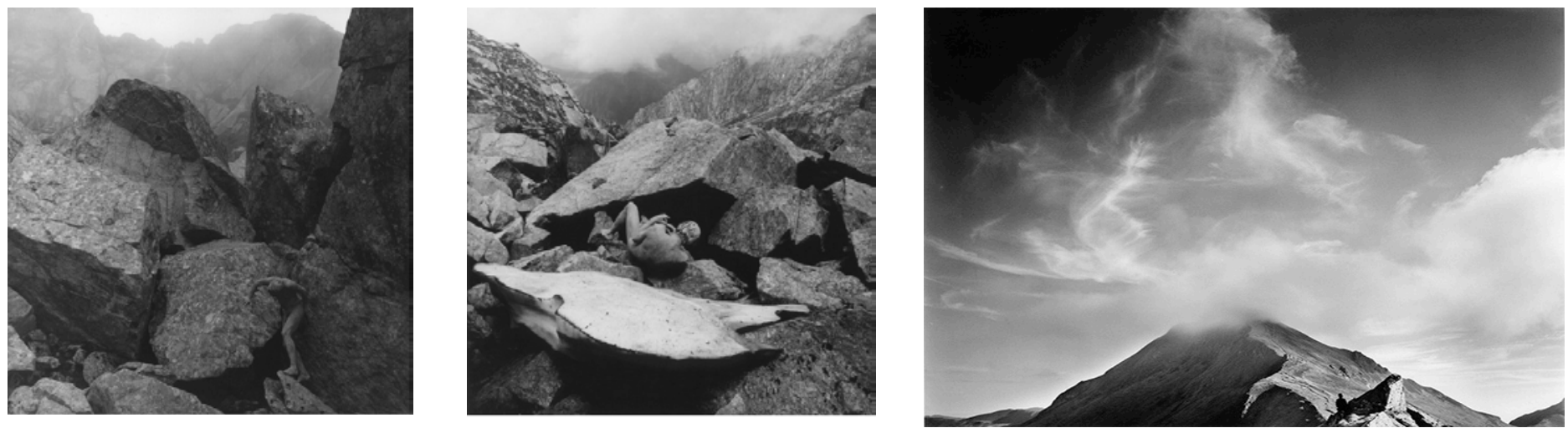

Minot \& Gormezano, Chaos VIII, 1985-86

Minot \& Gormezano, Le haut pays V, 2004

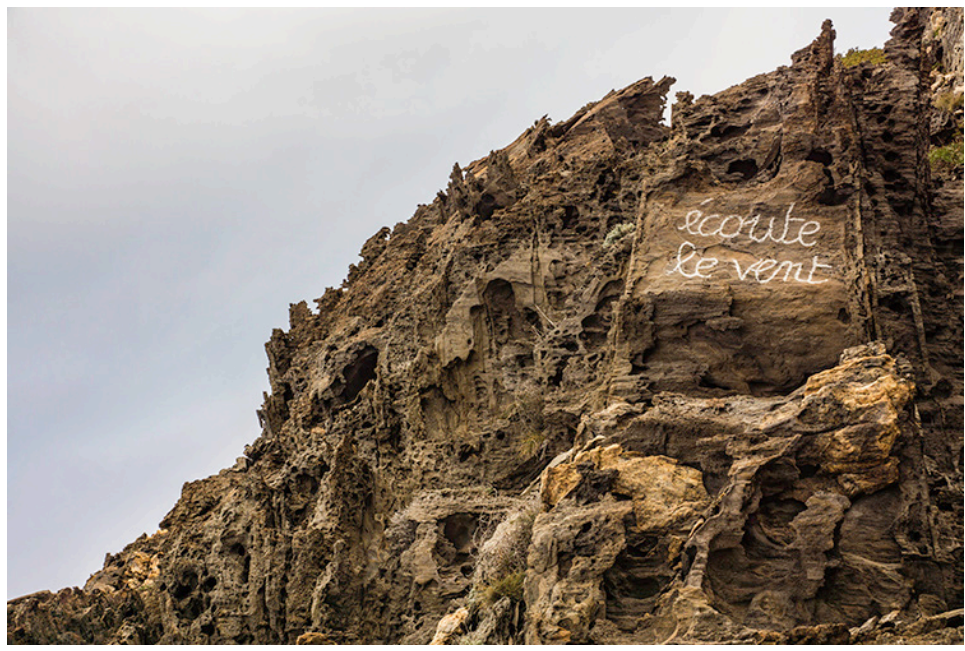

Minot \& Gormezano, Écoute le vent, série «Inscriptions », 2012.

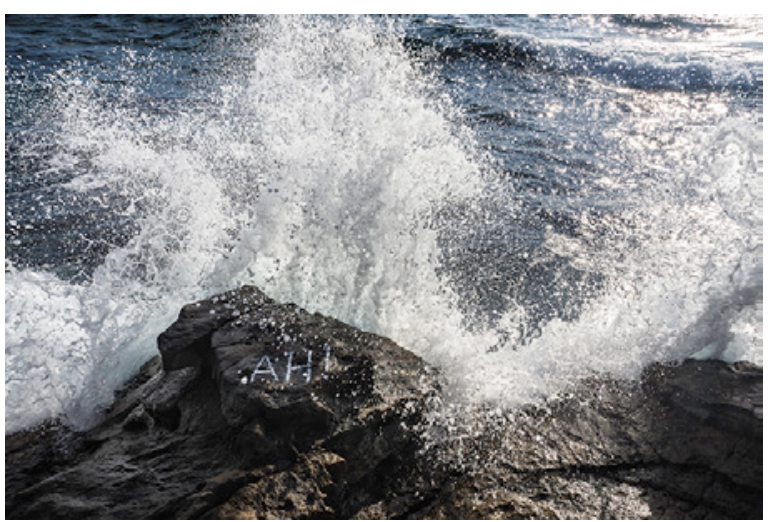

Minot \& Gormezano, Ah ! série «Inscriptions », 2014

22 http://www.minot-gormezano.com/

23 séries « Antres », « Chaos », « Le Cap », « Limons », « Vestiges »...

24 Séries « La mémoire hante le ciel », « Prélude aux métamorphoses », « Métamorphoses »

25 Séries « Terres de ciel », « Lieux », « Rêves », « Les passeurs »

26 « Les passeurs », « Inscriptions », « Recueils de voyages » (2012-2015)

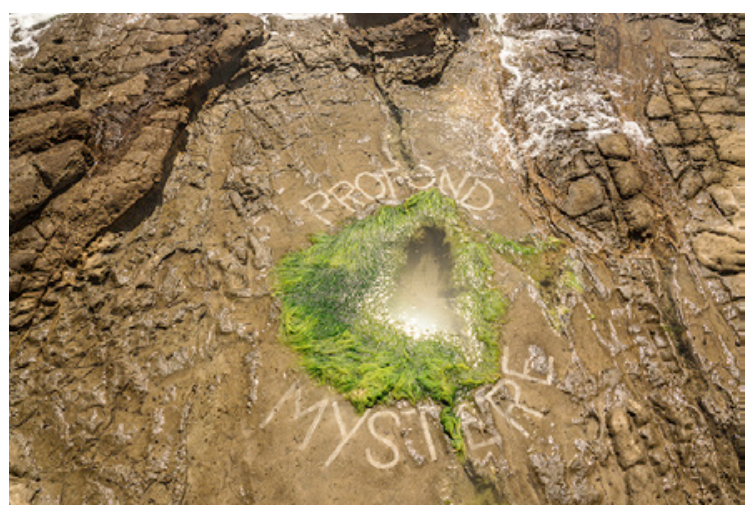

Minot \& Gormezano, Profond mystère, série «Inscriptions », 2012. 
Cette émergence du paysage de la relation entre le corps et le milieu arpenté est également au cœur des recherches d'Agnès Prévost ${ }^{27}$, pour son livre d'artiste Jocou, Corps, Va, qui est le premier volume de la collection « médiances », ainsi nommée en référence à la théorie mésologique d’Augustin Berque ${ }^{28}$. Ce livre aux pages découpées associe une photographie en noir et blanc et un texte pour raconter l'expérience vécue par l'artiste lors d'une marche en montagne, vers le Mont Jocou. L'ensemble exprime les qualités non seulement visibles mais aussi physiques et émotionnelles de cette relation entre le corps humain et le milieu traversé, dans sa relation avec la montagne, les roches, le vent... Dans ce livre d'artiste, la photographie paysagère sert de support au texte et le prolonge (je suis par exemple marquée par la correspondance entre l'image et ces quelques mots « entre les plis de la terre, tourne le souffle »). Elle nous invite à emboîter le pas de l'artiste et aiguiser nos sens pour mieux prendre conscience des médiances par lesquelles s’instaurent nos paysages.
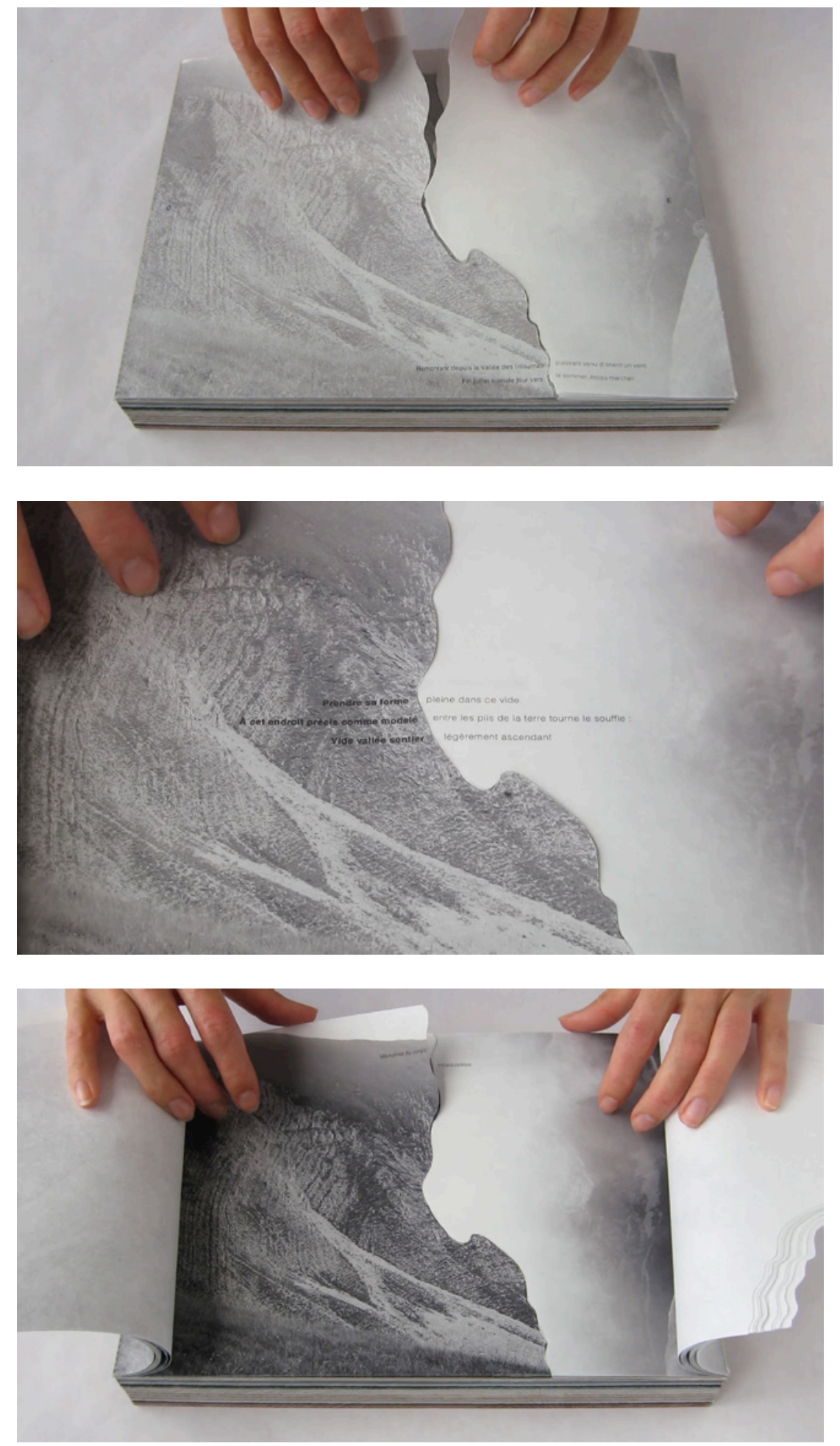

Agnès Prévost, Jocou, Corps, Va, livre d’artiste, collection « Médiances », 2018 


\section{Écopoétique paysagère : une expérience de création-recherche}

Mon propre travail photographique est mené dans le cadre de mes recherches sur une écopoétique paysagère. La préparation d'une thèse en arts plastiques sous les directions de Patrick Barrès et Sophie Lécole Solnychkine, selon la méthodologie de création-recherche propre à notre laboratoire, le LARA-SEPPIA ${ }^{29}$, m’a mené à développer une expérimentation artistique sur le terrain ${ }^{30}$. Dans le cadre d'une résidence artistique, j'ai photographié pendant deux années le site de Motten Morvan, un terrain de trois hectares en milieu rural, dans le centre de la Bretagne.

Ce travail se situe dans le prolongement des nouvelles approches du paysage chez les photographes français contemporains étudiées ci-dessus. Sur le plan théorique, mon approche de la photographie paysagère est inspirée par une réflexion sur l'écologie et ses représentations, m’ayant mené d'une réflexion critique sur la fiction verte à l'étude d'une piste alternative (esthétique et éthique) dans la construction d'une vision du monde écologique, sous la forme de l'écopoétique. Sur le plan artistique, ma pratique photographique est basée sur l'observation du paysage et intègre les arts du paysage, qu'il s'agisse de représentation visuelle, d'aménagement paysager, d'installation artistique in situ ou d'autres pratiques comme les promenades d'artistes. L'écriture de cet article m’a fait prendre conscience des singularités de la photographie française en particulier, et de son influence sur ma pratique. Le mode opératoire des Observatoires de paysage se retrouve en effet dans mon utilisation de la photographie comme moyen de notation dans le cadre d'une enquête paysagère, dans mon intérêt pour les paysages ordinaires, qui sont des territoires habités et façonnés par les activités humaines, et leur évolution dans le temps, à travers une esthétique de la sobriété plutôt que du spectaculaire. L'écologie scientifique a accompagné ma perception des paysages à travers les concepts d'écosystème et d'écocomplexe, et la manière dont ils évoluent en fonction (et en influant sur) des relations complexes entre les différents êtres

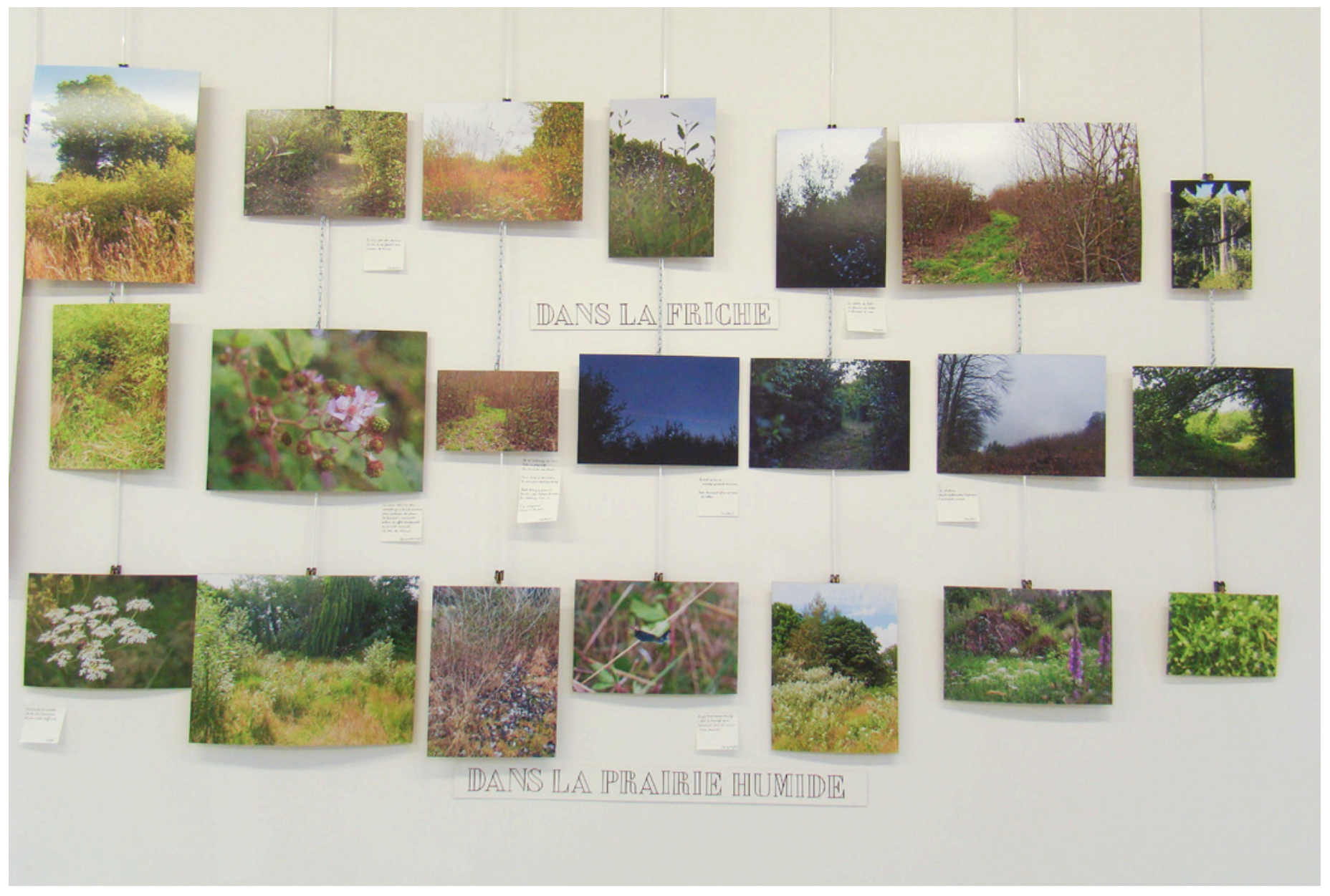

Anaïs Belchun, séries « Dans la friche » et « Dans la prairie humide », Morbihan, 2016-2018

(exposition « Dans les paysages de Motten Morvan », Pontivy, 2019)

29 https://lara.univ-tlse2.fr/

30 https://anais-belchun.wixsite.com/art-paysage-ecologie/projet-de-creation 


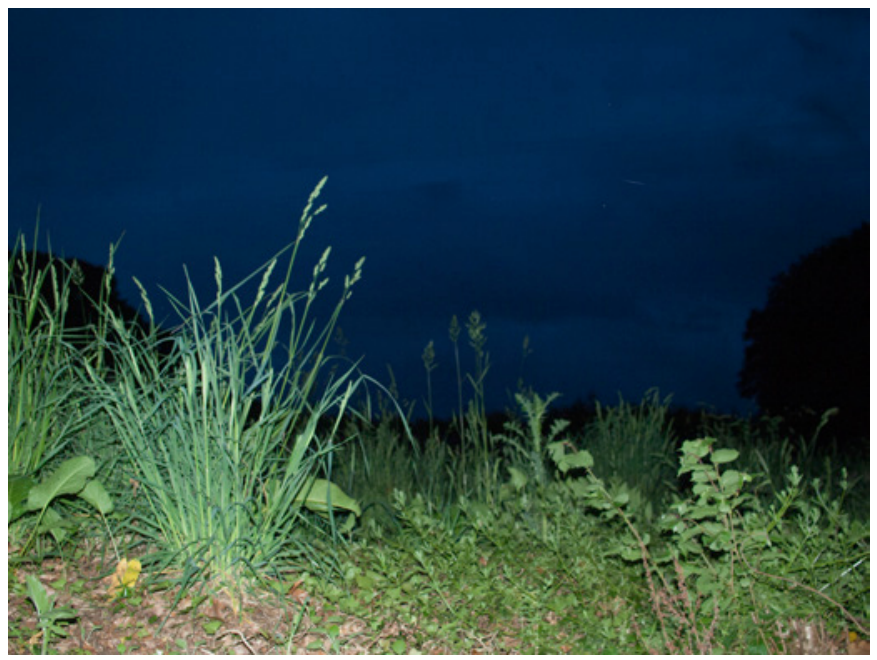

«Il s'agissait bien de l'herbe. Comment le sol insignifiant pouvait-il acquérir une telle densité, se manifester en somme par sa population et non par sa consistance? Je marchais sur une population. Les herbes. » (Gilles Clément)

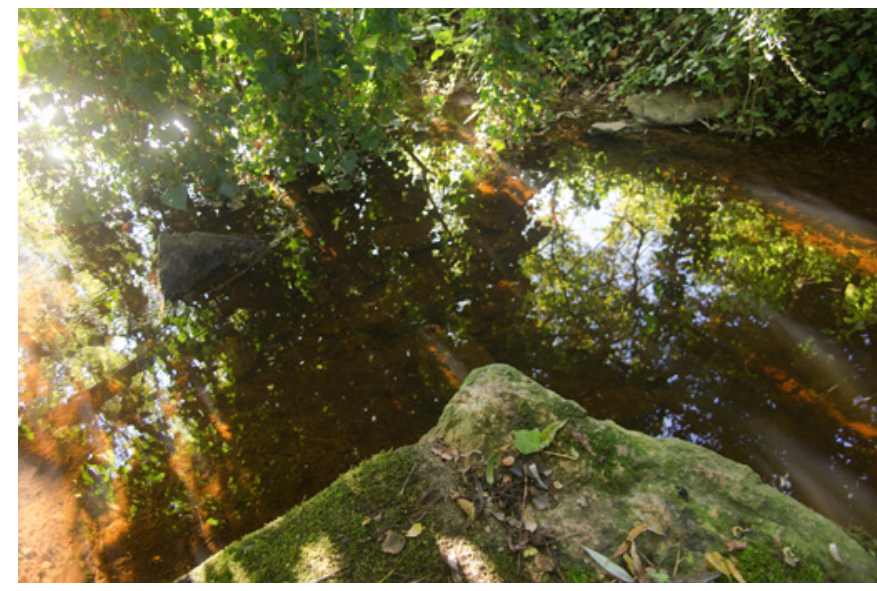

« Entre le roc et la feuille

Entre l'ombre et le soleil

Il y a des lois

Des lieux et des rites »

(Guillevic)

vivants (végétaux, animaux et humains), et les éléments et processus naturels, tout en portant attention aux singularités de chaque milieu en fonction de la situation et des dynamiques locales, avec un intérêt particulier pour les friches. Les photographies plus artistiques ont nourri ma recherche d'une approche sensible du paysage à travers l'expérience vécue (avec un intérêt marqué pour les perceptions sensorielle, la sensibilité émotionnelle et esthétique, et la mise en œuvre de processus de signification). Ma pratique de la photographie se fonde donc sur l'expérience sensible, à travers un processus immersif et dans la durée, qui s'exprime dans la variation des sujets et des conditions de prise de vue, mais aussi la diversité de points de vue, focales, perspectives...

La première phase de ce travail de création-recherche a pris la forme d'une enquête paysagère, associant expérience sensible, étude documentaire, lecture de paysage et notation paysagère, dans laquelle la prise de vue photographique joua un rôle important. Une centaine de ces photographies ont ensuite été présentées dans une exposition intitulée « Dans les paysages de Motten Morvan ». Elles ont été disposées en six séries, de manière à présenter les différents ensembles écosystémiques et paysagers identifiés sur le site : la prairie, la forêt, la friche, la cour de ferme, la prairie humide et le bord du ruisseau. Le choix de ces photographies a été effectué de manière à proposer un regard écopoétique sur ces différents milieux, en reflétant la diversité des expériences paysagères que j’ai vécues sur ce site. Chaque milieu est présenté sous différents points de vue (immersion, vue d'ensemble, gros plans au plus proche de la matière et du vivant), à différentes périodes de l'année (au moins une image de chaque saison), à différentes heures du jour et de la nuit (au moins une photo de nuit), selon différentes situations météorologiques (ciel bleu ou nuageux, soleil, pluie, gel, sécheresse...). Une attention particulière est portée au vivant, avec dans chaque milieu une sélection de plantes ou d'insectes mettant en valeur la beauté de chaque être, et particulièrement des espèces communes habituellement négligées. De courts textes sont associés à certaines photographies ; il s’agit de citations extraites d'œuvres de littérature écopoétique (Guillevic, Gary Snyder, Élisée Reclus, Aldo Leopold, Henry David Thoreau, David Haskell, Gilles Clément, Bashô et autres auteurs de haïkus).

L’organisation spatiale de cette scénographie est une retranscription hors du site de deux éléments de mon enquête paysagère. D’une part, elle fait écho aux paramètres écologiques repérés en référence aux concepts issus de l'écologie scientifique, notamment de l'écologie du paysage. On retrouve dans ces photographies les différents écosystèmes identifiés sur le terrain, mais aussi les paramètres éco-paysagers propres à ce site, et caractéristiques de cette région du centre de la Bretagne : une diversité biologique et écosystémique importante, une hétérogénéité paysagère remarquable, une faible connectivité et une complexité moyenne. D’autre part, elle reproduit le parcours que j'effectuais sur le terrain, passant d'un milieu à un autre au cours de la marche, et donc retranscrit l'expérience sensible vécue sur le terrain. Le spectateur, en marchant le long du mur où sont exposées les photographies qu’il observe, est ainsi invité à ré-inventer ce scénario paysager en adoptant un nouveau regard sur ces paysages ordinaires. 